\title{
Aldol condensations in pyrazino[2,1-b]quinazolines. Fast access to seco-ardeemin derivatives
}

\author{
Pilar Cledera, Mercedes Villacampa, Carmen Avendaño, and J. Carlos Menéndez* \\ Departamento de Química Orgánica y Farmacéutica, Facultad de Farmacia, Universidad \\ Complutense, 28040 Madrid, Spain \\ E-mail:josecm@farm.ucm.es
}

\begin{abstract}
This paper is dedicated to Professor Julio Álvarez-Builla, on the occasion of his $65^{\text {th }}$ birthday
\end{abstract}

\begin{abstract}
The reaction between 2-alkylpyrazino[2,1-b]quinazoline-3,6-diones and aromatic aldehydes in the presence of $\mathrm{KO}^{\mathrm{t}} \mathrm{Bu}$ afforded the corresponding 1-arylmethylene derivatives, preferently as the $Z$ isomers. This diastereoselectivity was the result of thermodynamic control, as shown by $a b$ initio calculations. These aldol reactions proceeded with partial racemization of the C-4 stereocenter, in contrast with the condensations of the related 2-acetylpyrazino[2,1b]quinazoline-3,6-diones. Catalytic hydrogenation of the 1-arylmethylene derivatives obtained through these condensations afforded cis-1,4-disubstituted pyrazino[2,1-b]quinazoline-3,6diones, normally with complete diastereoselectivity, but these reactions had compounds from partial reduction of the benzene ring as side products.
\end{abstract}

Keywords: Aldol condensation, pyrazino[2,1-b]quinazolines, diastereoselectivity, catalytic hydrogenation, benzene hydrogenation

\section{Introduction}

The quinazolin-4-one family of alkaloids ${ }^{1}$ comprises about 150 compounds that have been isolated from fungi, marine organisms and higher plants. Benzo-fused derivatives of this system are also common in nature, the most important ones being pyrazino[2,1-b]quinazoline-3,6diones. ${ }^{2}$ This system is the key structural fragment of a group of fungal metabolites which often exhibit very interesting biological properties. The simplest member of this family of alkaloids is glyantrypine, isolated from Aspergillus clavatus. ${ }^{3} \mathrm{C}_{4}$-substituted compounds include members of the fumiquinazoline ${ }^{4}$ and fiscalin ${ }^{5}$ families, some of which have shown cytotoxicity and antifungal activities. More complex systems include $N$-acetylardeemin, which was isolated from 
Aspergillus fischeri ${ }^{6 \mathrm{a}}$ and is one of the most potent known inhibitors ${ }^{7}$ of multi-drug resistance to antitumour compounds (MDR), which can be considered as the most important single factor that prevents the success of antitumour chemotherapy in many cancer patients. ${ }^{8}$ Thus, $\mathrm{N}$ acetylardeemin potentiated the cytotoxicity of vinblastine, doxorubicin or paclitaxel in multidrug resistant human tumor cells, with a 10 -fold potency with respect to verapamil, the standard antiMDR compound. Its synthetic glycine analog and its trifluoro derivative are also potent MDR reversal agents that bind to the two main transport proteins responsible the MDR phenomenon, namely P-gp 170 and MRP. ${ }^{6 b, 9}$ The study of simplified ardeemin analogues has led to the conclusion that the pyrazino[2,1-b]quinazoline fragment can be considered as its pharmacophoric moiety for MDR reversal activity, which underscores the importance of this tricyclic system. ${ }^{10}$ Another interesting property of some members of the ardeemin family is their immunosupressant activity. ${ }^{11}$
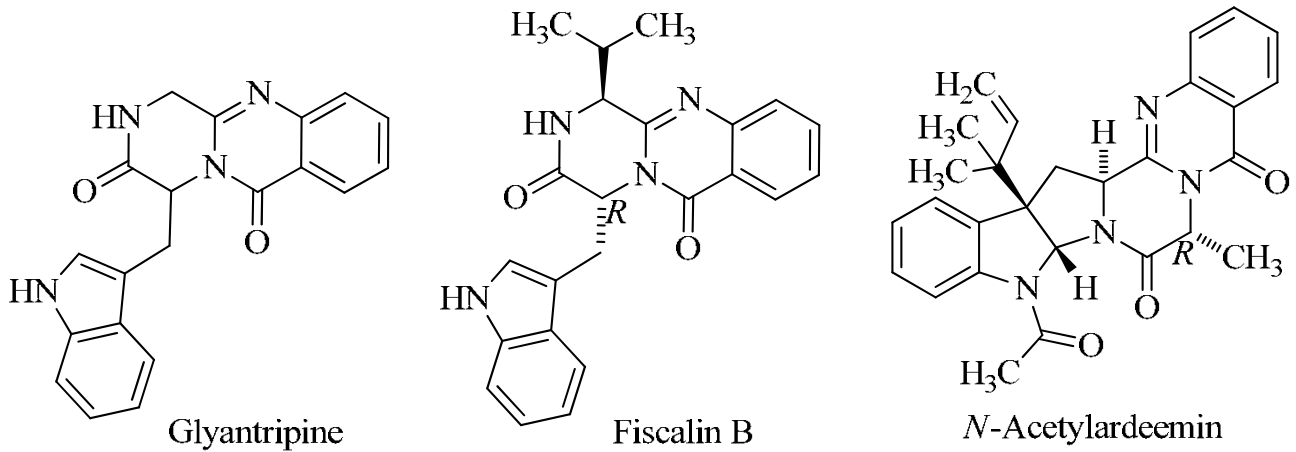

Figure 1. Representative pyrazino[2,1-b]quinazoline alkaloids.

\section{Results and Discussion}

Due to the pharmacological interest of pyrazino[2,1-b]quinazolines as MDR modulators, ${ }^{10 a}$ we became interested in 1-arylmethylene derivatives of the parent system 1, which can be considered as seco-analogues of ardeemin. Since MDR reversal activity normally correlates well with lipophilicity, ${ }^{8}$ we designed compounds $\mathbf{1}$ to be $N$-substituted. Compounds $\mathbf{1}$ where $\mathrm{R}^{2}=\mathrm{H}$ have been obtained by von Niementowsky or aza Wittig reactions from 6-arylmethylene-2,5piperazinediones, ${ }^{12}$ which were in turn obtained by base-catalyzed condensation of 1,4-diacetyl2,5-piperazinedione with aromatic aldehydes, a reaction that takes place with anchimeric assistance from the $\mathrm{N}$-acetyl substituent and is accompanied by $\mathrm{N}$-deacetylation. Since regioselective $N$-alkylation is difficult due to the steric hindrance caused by the arylmethylene substituent and may be hampered by competing deprotonation at C-4, the most direct approach to our target compounds $\mathbf{1}$ would be one based on the direct aldol condensation of compounds $\mathbf{2}$ with aromatic aldehydes. These starting materials were known compounds, ${ }^{12 b, 13}$ but we have developed an improved preparation of $\mathbf{2 a}$ based on an acylation/aza-Wittig strategy (see 
Experimental). Furthermore, the availability of $\mathbf{1}$ would allow the simple preparation of compounds $\mathbf{3}$ in an alternative approach to the one involving the base-promoted alkylation of $2{ }^{14}$ which has disadvantages such as lack of diastereoselectivity and the isolation of double alkylation products (Scheme 1). The cis-compounds $\mathbf{3}$ are interesting in spite of having the opposite relative configuration to ardeemin because it has been shown for another family of simplified analogues of the ardeemin ABCD ring system that compounds with an opposite configuration to the natural product at the alanine stereocenter had improved activity as MDR reversors. ${ }^{10 \mathrm{~b}}$<smiles>C=CC1C(=O)N(C(=O)Cl)CC(=O)N1C(C)(C)Cl</smiles><smiles>C=CC1NC(=O)C(=C[Al])NC1=O</smiles><smiles>[R]N1C(=O)C(C)n2c(nc3ccccc3c2=O)C1=C[Al]</smiles><smiles>C=CCC</smiles><smiles>[R]N1Cc2nc3ccccc3c(=O)n2C(C)C1=O</smiles>

2<smiles>[Y7]C[C@H]1c2nc3ccccc3c(=O)n2[C@@H](C)C(=O)N1[R]</smiles>

\section{Scheme 1}

The results of the systematic study ${ }^{15}$ of the condensation of compounds 2a-c with several aromatic aldehydes derived from benzaldehyde and indole-3-carbaldehyde in the presence of a slight excess of potassium tert-butoxide $\left(\mathrm{KO}^{\mathrm{t}} \mathrm{Bu}\right)$ are shown in Scheme 2 and Table 1 . The assignment of the $Z$ configuration to the major products was based on the chemical shift of the vinylic proton, which was observed at $c a$. $7.40 \mathrm{ppm}$ due to a deshielding effect caused by the neighbouring $\mathrm{C}=\mathrm{N}$ group, while for the $E$ isomers this signal was observed at about $6.70 \mathrm{ppm}$. This assignment was confirmed by NOE effects in representative compounds. 


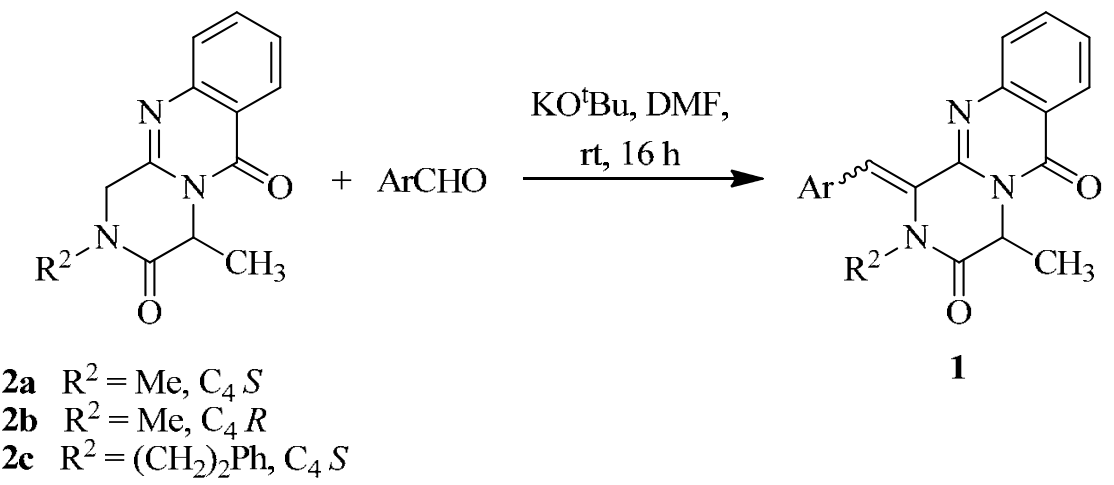

\section{Scheme 2}

Table 1. Scope and yields of the reaction between compounds 2 and aldehydes

\begin{tabular}{ccccccc}
\hline Entry & $\begin{array}{c}\text { Starting } \\
\text { material }\end{array}$ & Product & $\mathrm{Ar}$ & $\mathrm{R}^{2}$ & $\begin{array}{c}\text { Yield of } \\
(Z)-\mathbf{1}, \%\end{array}$ & $\begin{array}{c}\text { Yield of } \\
(E)-\mathbf{1}, \%\end{array}$ \\
\hline 1 & $\mathbf{2 a}$ & $\mathbf{1 a}$ & $4-\mathrm{ClC}_{6} \mathrm{H}_{4}$ & $(S)-\mathrm{CH}_{3}$ & 73 & 26 \\
2 & $\mathbf{2 b}$ & $\mathbf{1 b}$ & $4-\mathrm{ClC}_{6} \mathrm{H}_{4}$ & $(R)-\mathrm{CH}_{3}$ & 75 & 24 \\
3 & $\mathbf{2 c}$ & $\mathbf{1 c}$ & $4-\mathrm{ClC}_{6} \mathrm{H}_{4}$ & $\mathrm{CH}_{2} \mathrm{CH}_{2} \mathrm{C}_{6} \mathrm{H}_{5}$ & 48 & 46 \\
4 & $\mathbf{2 a}$ & $\mathbf{1 d}$ & $4-\mathrm{BrC}_{6} \mathrm{H}_{4}$ & $\mathrm{CH}_{3}$ & 45 & 22 \\
5 & $\mathbf{2 a}$ & $\mathbf{1 e}$ & $4-\mathrm{NO}_{2} \mathrm{C}_{6} \mathrm{H}_{4}$ & $\mathrm{CH}_{3}$ & 71 & $3^{\mathrm{a}}$ \\
6 & $\mathbf{2 a}$ & $\mathbf{1 f}$ & $3,4-\mathrm{Cl}_{2} \mathrm{C}_{6} \mathrm{H}_{3}$ & $\mathrm{CH}_{3}$ & 62 & 23 \\
7 & $\mathbf{2 a}$ & $\mathbf{1 g}$ & $2,4-\mathrm{F}_{2} \mathrm{C}_{6} \mathrm{H}_{3}$ & $\mathrm{CH}_{3}$ & 87 & 0 \\
8 & $\mathbf{2 a}$ & $\mathbf{1 h}$ & 1-Benzyl-3-indolyl & $\mathrm{CH}_{3}$ & 53 & $38^{\mathrm{b}}$ \\
9 & $\mathbf{2 a}$ & $\mathbf{1 i}$ & 1-Tosyl-3-indolyl & $\mathrm{CH}_{3}$ & 36 & 16 \\
19 & $\mathbf{2 a}$ & $\mathbf{1 j}$ & 4-MeOC $6 \mathrm{H}_{4}$ & $\mathrm{H}$ & 63 & $16^{\mathrm{c}}$ \\
\hline
\end{tabular}

${ }^{\mathrm{a}}$ Together with $10 \%$ of $4 .{ }^{\mathrm{b}}$ Measured by NMR of the crude reaction product. ${ }^{\mathrm{c}}$ Together with $20 \%$ of 5 .

Some of the condensation reactions afforded significant amounts of side products. In the case of the reaction between 2a and 4-nitrobenzaldehyde, we isolated the 1-oxo derivative of the starting material (compound 4). As shown in Scheme 3, the formation of $\mathbf{4}$ is probably due to the fact that the C-1 position of 2a allows the generation of a radical stabilized by captodative effect ${ }^{16}$ (intermediate 6 ), which can then react with a molecule of oxygen ${ }^{17}$ to give 7 . Attack of this radical to a fresh molecule of 2a regenerates $\mathbf{6}$, closing the propagation cycle, and affords hydroperoxide 8. Finally, loss of a molecule of water from $\mathbf{8}$ explains the formation of the observed product 4 . The fact that this side reaction was only observed in the reaction with 4nitrobenzaldehyde may be related to the ability of nitro groups to act as intermediates in oneelectron transfer reactions. ${ }^{18}$ This observation leads us to favour the explanation summarized in Scheme 3 over an alternative one based on the reaction of an anion at C-1 followed by reaction 
with oxygen. Radical formation at C-4 would also be possible, in principle, but it would lead to racemization, which has not been observed.

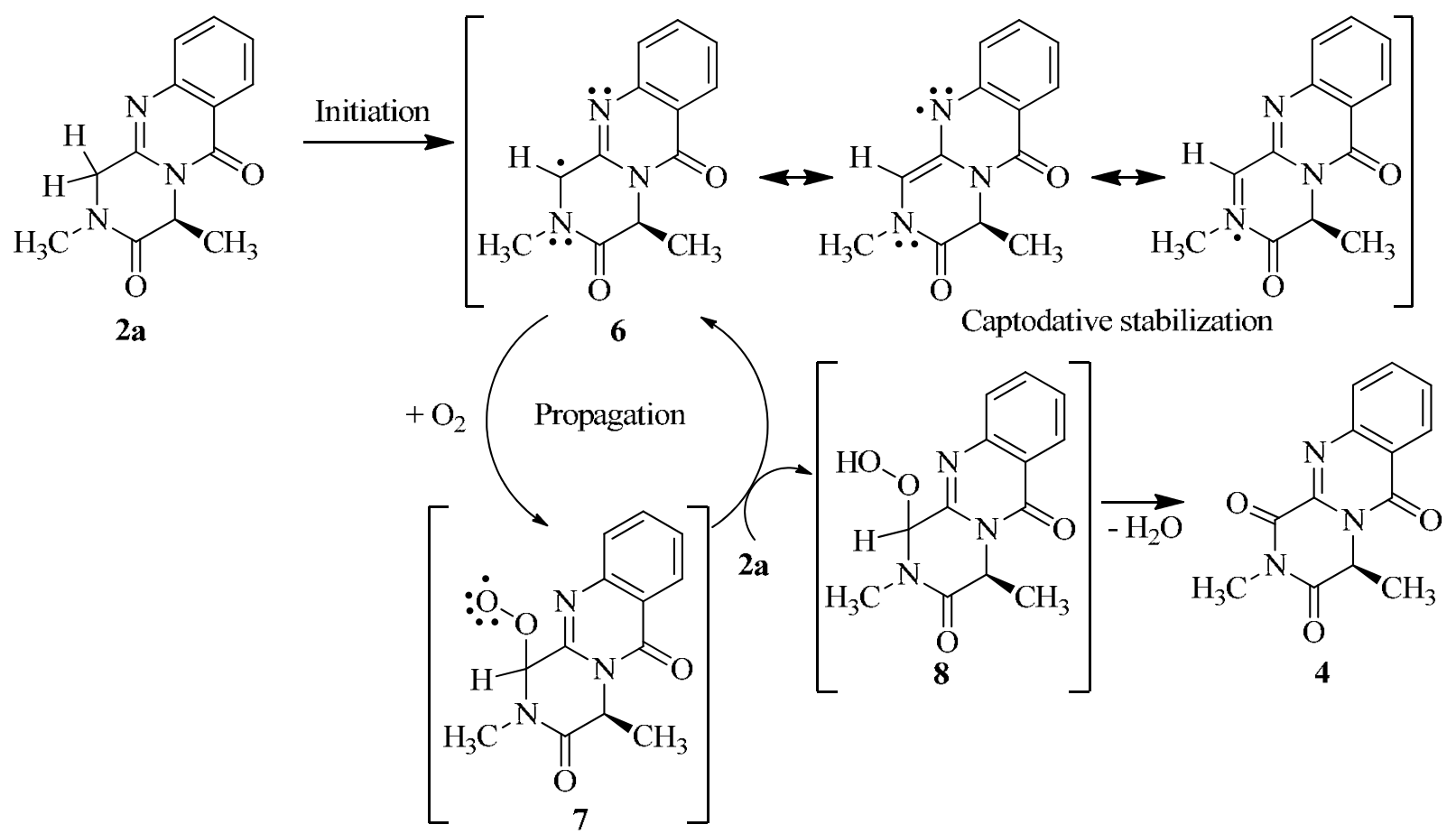

\section{Scheme 3}

Another unexpected product was obtained in the reaction between 2a and 4methoxybenzaldehyde, in which case compound $\mathbf{1 j}$ was accompanied by compound $\mathbf{5}$ in 1.5:1 $Z / E$ ratio. The formation of $\mathbf{5}$ can be explained by the formation of the dipole-stabilized carbanion $^{19} 9$ by deprotonation of the $N$-methyl carboxamide portion in the initial reaction product $\mathbf{1 j}$. The reaction of $\mathbf{9}$ with the starting aldehyde, followed by oxidation, explains the formation of 5 (Scheme 4). In agreement with this explanation, only traces of $\mathbf{5}$ were isolated when the reactions were carried out in the presence of an equimolecular amount of base. The low $Z / E$ diastereoselectivity observed for 5 agrees with the one observed for the $N$-phenetyl derivative 1c (ca. 1:1), which was much lower than that found for $N$-methyl derivatives. This difference is discussed below (see table 3, Figure 2 and accompanying text). 


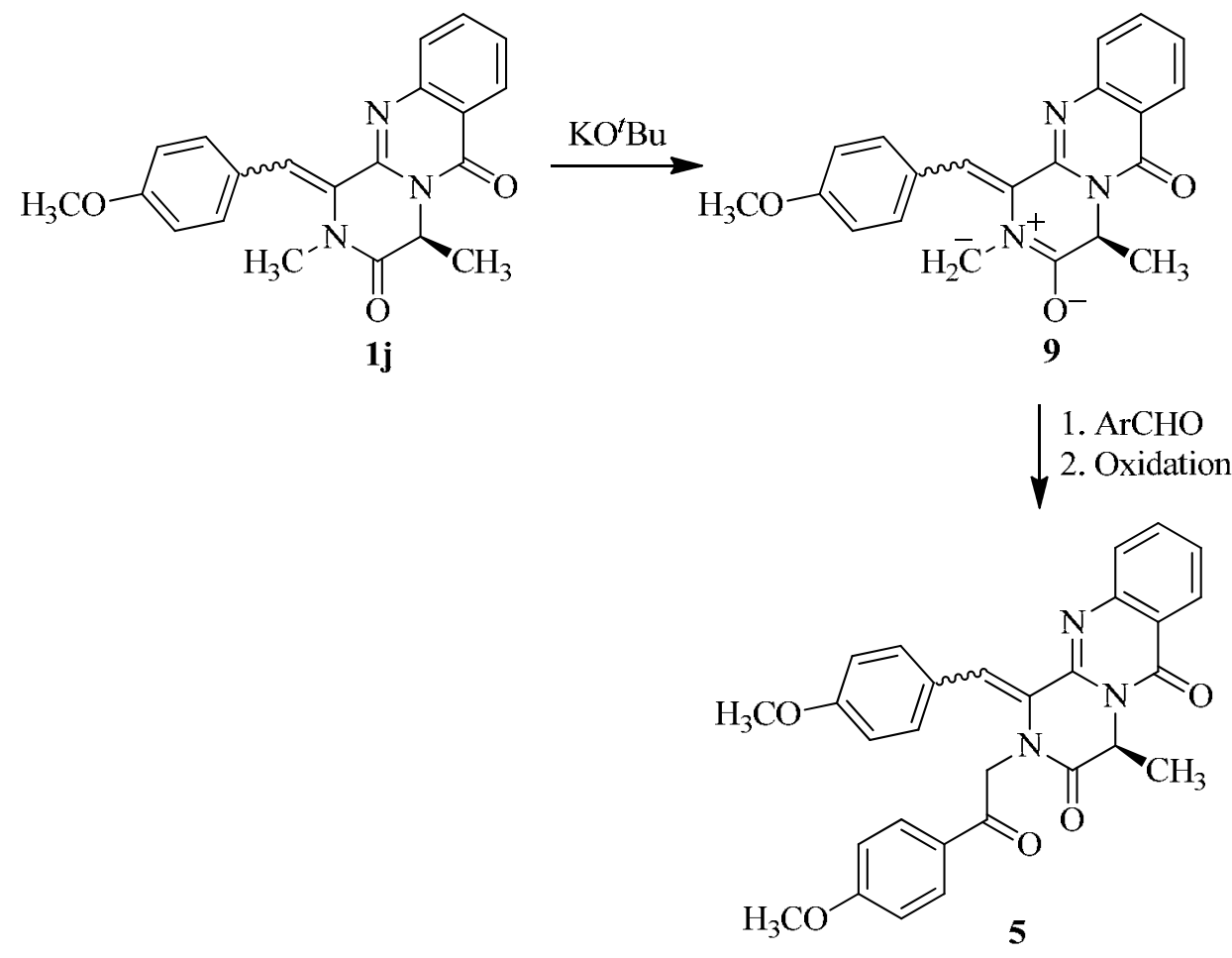

\section{Scheme 4}

In order to increase the synthetic scope of our reaction, we decided to study the related process starting from $\mathrm{N}$-acetyl derivatives of the starting material. This transformation is related to the analogous reaction between $N$-acetyl-2,5-piperazinediones and aldehydes, which is well precedented in the literature and is known to be accompanied by $N$-deacetylation and to lead exclusively to products with $Z$ configuration. As starting material, we selected compound 2d, which is available through a method developed during our studies on the total synthesis of glyantripine. ${ }^{3 b}$ According to the literature precedent on the simpler piperazinedione systems, treatment of $\mathbf{2 d}$ with 4-chlorobenzaldehyde in the presence of $\mathrm{KO}^{t} \mathrm{Bu}$ at room temperature afforded an excellent yield of the deacetylated condensation product $\mathbf{1 k}$, which was isolated exclusively as the $Z$ isomer. The mechanism proposed for this transformation is summarized in Scheme 5, and is based on the one commonly accepted for the related reaction of $N$-acetyl-2,5piperazinediones. $^{20}$ 


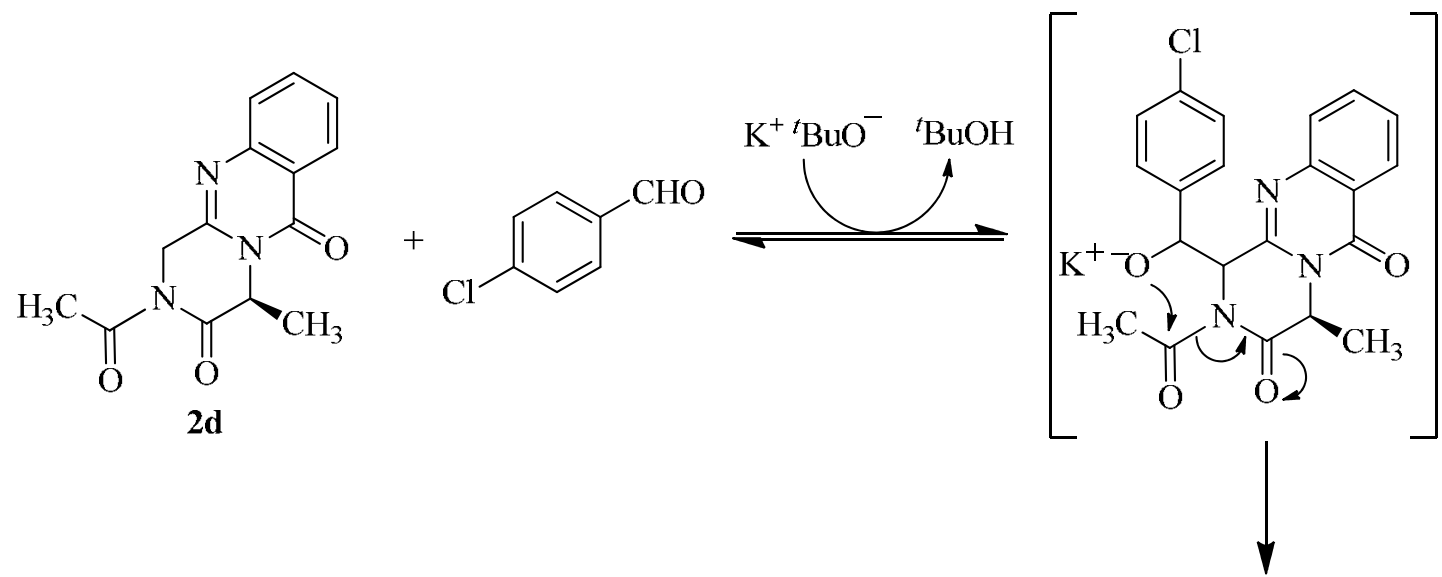

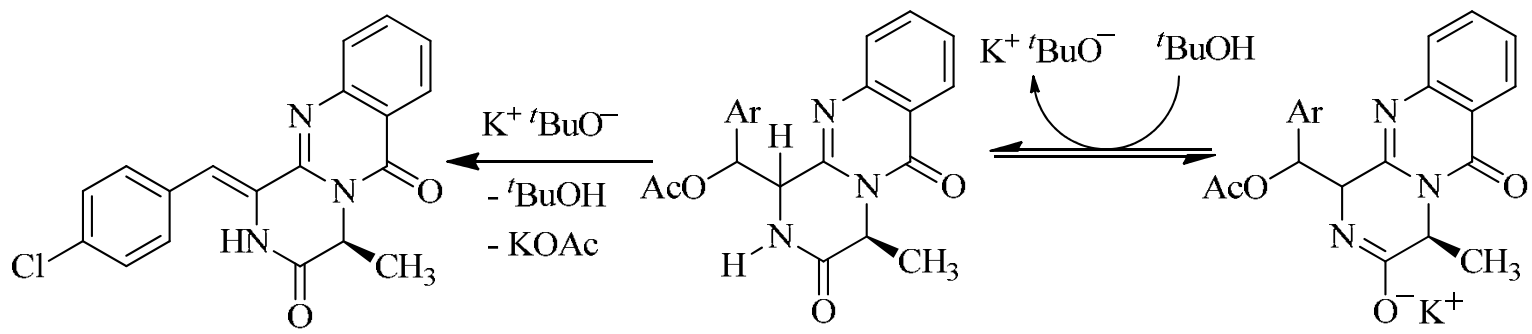
$1 k(94 \%)$

\section{Scheme 5}

Reactions involving the use of a strong base can potentially affect the stereocenter adjacent to the carbonyl. When compounds $\mathbf{1 a}$ - $\mathbf{j}$ were examined using either ${ }^{1} \mathrm{H}-\mathrm{NMR}$ in the presence of $(+)$ europium tris[3-(trifluoromethylhydroxymethylene)]camphorate (Eu(hfc) $)_{3}$ or by HPLC using a cellulose carbamate chiral column, enantiomeric ratios were normally poor (Table 2), although this will not probably constitute a problem in terms of bioactivity, since MDR inhibition is not normally stereospecific. ${ }^{8}$ Replacement of $\mathrm{KO}^{t} \mathrm{Bu}$ by cesium carbonate, a much weaker base, did not improve this result, as verified for the case of compound 1a. Comparison of the results summarized in Tables 1 and 2 suggests a correlation between $Z: E$ and enantiomeric ratios, since reactions with better $Z$ selectivity are also more enantioselective (compare entries 5 and 7 of both Tables with the other results). On the other hand, the $N$-unsubstituted compound $1 \mathbf{k}$ was obtained as a single enantiomer as in the case of aldol condensations starting from (S)-1,4-diacetyl-3methyl-2,5-piperazinedione, using $\mathrm{KO}^{\mathrm{t}} \mathrm{Bu}$ as a base, in which the stereocenter maintains its integrity within the limits of detection of ${ }^{1} \mathrm{H}-\mathrm{NMR}$ in the presence of a chiral shift reagent. ${ }^{12 a}{ }^{21}$, This behaviour may be explained by considering that an aldol reaction at the position adjacent to a $\mathrm{N}$-acetyl susbtituent is accompanied by its deacetylation, which liberates an ionizable $\mathrm{NH}$ group at the end of the process that hampers a second deprotonation at the stereocenter. In the case of compound $\mathbf{1 k}$, the absence of racemization is probably due to the existence of a free $\mathrm{NH}$ group that makes more probable the formation of a conjugated anion $\mathbf{1 0}$ rather than 11, which is the one required for the inversion of the stereocenter (Scheme 6). 
Table 2. Enantiomeric ratios found for the $Z$ isomers of compounds 1

\begin{tabular}{|c|c|c|c|c|c|}
\hline Entry & Compound & $\mathrm{Ar}$ & $\mathrm{R}^{2}$ & e.r. & Technique \\
\hline 1 & 1a & $4-\mathrm{ClC}_{6} \mathrm{H}_{4}$ & $\mathrm{CH}_{3}$ & $2: 1$ & $\begin{array}{c}\text { HPLC } \\
{ }^{1} \mathrm{H}-\mathrm{NMR}\end{array}$ \\
\hline 2 & 1b & $4-\mathrm{ClC}_{6} \mathrm{H}_{4}$ & $\mathrm{CH}_{3}$ & $2: 1$ & $\begin{array}{c}\text { HPLC } \\
{ }^{1} \mathrm{H}-\mathrm{NMR}\end{array}$ \\
\hline 3 & 1c & $4-\mathrm{ClC}_{6} \mathrm{H}_{4}$ & $\mathrm{CH}_{2} \mathrm{CH}_{2} \mathrm{C}_{6} \mathrm{H}_{5}$ & $1: 1$ & $\begin{array}{c}\text { HPLC } \\
{ }^{1} \mathrm{H}-\mathrm{NMR}\end{array}$ \\
\hline 4 & 1d & 4- $\mathrm{BrC}_{6} \mathrm{H}_{4}$ & $\mathrm{CH}_{3}$ & $3: 1$ & ${ }^{1} \mathrm{H}-\mathrm{NMR}$ \\
\hline 5 & 1e & $4-\mathrm{NO}_{2} \mathrm{C}_{6} \mathrm{H}_{4}$ & $\mathrm{CH}_{3}$ & $8: 1$ & $\begin{array}{c}\text { HPLC } \\
{ }^{1} \mathrm{H}-\mathrm{NMR}\end{array}$ \\
\hline 6 & 1f & $3,4-\mathrm{Cl}_{2} \mathrm{C}_{6} \mathrm{H}_{3}$ & $\mathrm{CH}_{3}$ & $1.5: 1$ & ${ }^{1} \mathrm{H}-\mathrm{NMR}$ \\
\hline 7 & $1 g$ & $2,4-\mathrm{F}_{2} \mathrm{C}_{6} \mathrm{H}_{3}$ & $\mathrm{CH}_{3}$ & $8: 1$ & $\begin{array}{c}\text { HPLC } \\
{ }^{1} \mathrm{H}-\mathrm{NMR}\end{array}$ \\
\hline 8 & $1 \mathrm{~h}$ & 1-Benzyl-3-indolyl & $\mathrm{CH}_{3}$ & $2: 1$ & ${ }^{1} \mathrm{H}-\mathrm{NMR}$ \\
\hline 9 & $\mathbf{1 j}$ & $4-\mathrm{MeOC}_{6} \mathrm{H}_{4}$ & $\mathrm{CH}_{3}$ & $1.5: 1$ & $\begin{array}{c}\text { HPLC } \\
{ }^{1} \mathrm{H}-\mathrm{NMR}\end{array}$ \\
\hline 10 & $1 \mathrm{k}$ & 4- $\mathrm{ClC}_{6} \mathrm{H}_{4}$ & $\mathrm{H}$ & $>98: 2$ & HPLC \\
\hline
\end{tabular}<smiles>C[C@H]1C([O-])=N/C(=C\[Al])c2nc3ccccc3c(=O)n21</smiles>

10<smiles>C[C@H]1C(=O)N/C(=C\[Al])c2nc3ccccc3c(=O)n21</smiles>

$1 \mathrm{k}$<smiles>C=CC=C</smiles><smiles>CC1=C([O-])N/C(=C\[Al])c2nc3ccccc3c(=O)n21</smiles>

11<smiles>[3H]/C=C1\NC(=O)[C@H](C)n2c1nc1ccccc1c2=O</smiles>

ent-1k (not observed)

\section{Scheme 6}

In order to allow a meaningful comparison between piperazinediones and compounds $\mathbf{1 a - j}$, we studied the aldol condensation of a $N$-substituted-2,5-piperazinedione, and to this end we prepared compound 13 by acetylation of the known ${ }^{22}$ cyclo-(sarcosine-L-alanine) 12. Treatment of 13 with 4-chlorobenzaldehyde under our usual conditions afforded a mixture of the $Z$ and $E$ condensation products 14 in low yields because of base-promoted deacetylation of the starting material. In contrast with our previous observations on compounds $\mathbf{1 a - j}$, the major product from this mixture was found to contain a single enantiomer by ${ }^{1} \mathrm{H}-\mathrm{NMR}$ in the presence of Eu(hfc) 3 . This difference in behavior can be attributed to fact that in the case of $\mathbf{1 4}$ the acidic proton responsible for the racemization is more hindered due to the conformational freedom of the 
neighbouring $\mathrm{N}$-acetyl substituent, whereas in the rigid compounds $\mathbf{1}$ this proton is more accessible (Scheme 7).

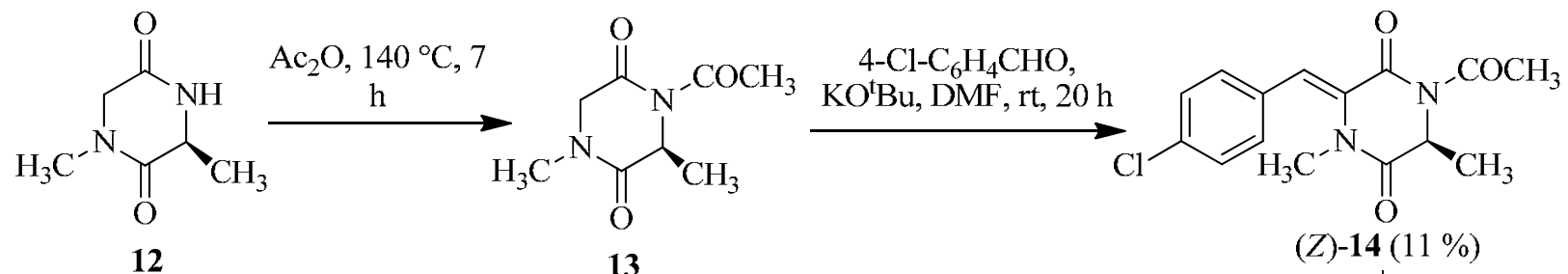

12

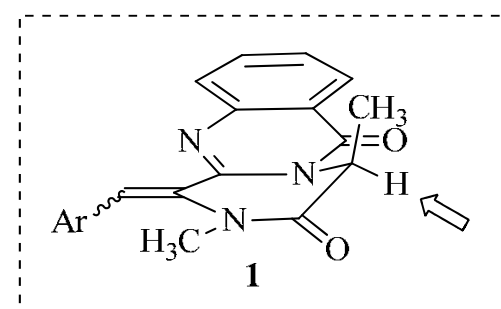

13

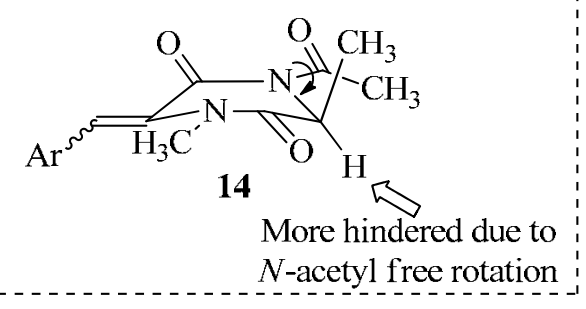

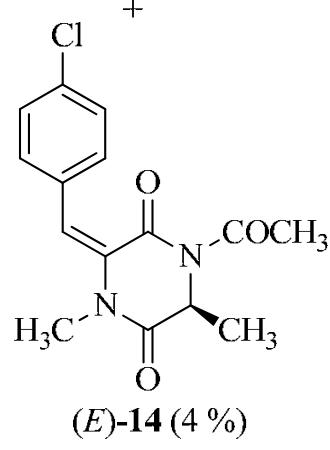

\section{Scheme 7}

The Z-selectivity of the aldol reactions was attributed to thermodynamic control, since the $Z$ isomers were found to be more stable according to $a b$ initio calculations at the HF 6-31G level. The results obtained are collected in Table 3, which shows that the differences in stability between the $Z$ and $E$ isomers correlate well with the experimental diastereomeric ratios. Thus, in the case of 1a, for which the experimental d.r. was $c a$. 3:1, the $Z$ isomer was more stable than the $E$ in $2.88 \mathrm{kcal}^{\mathrm{mol}}{ }^{-1}$ (entry 1). This difference was only $0.92 \mathrm{kcal}^{\mathrm{mol}}{ }^{-1}$ in case of the $N$ phenethyl derivative 1c (entry 2), where the $Z$ and $E$ isomers were isolated in equimolecular amounts, probably due to an increased repulsion between the bulkier $N$-substituent and the arylmethylene side chain. The calculated differences in stability were much higher (ca. 6 kcal.mol ${ }^{-1}$ ) for the $N$-unsubstituted compounds $1 \mathbf{k}$ and $\mathbf{1 4}$, for which the $E$ isomer was not observed (entries 3 and 4). As shown in Figure 2 for the $a b$ initio-minimized structures, the lower stability of the $E$ isomers can be attributed to repulsive interactions between the C-1 and C-4 substituents. In the case of compound $(E)-\mathbf{1 b}$, having an $R$ configuration at $\mathrm{C}-4$, this interaction cannot take place. In this case, the conformation of the piperazine ring changes to the other possible boat structure in order to avoid the repulsive interaction between the $\mathrm{C}_{4}$-methyl and $\mathrm{C}_{6}=\mathrm{O}$ groups, and this brings the arylmethylene unit close to the tricyclic system, explaining the lower stability of this compound compared with its $(Z)$ isomer. 
Table 3. Calculated energy differences between the $Z$ and $E$ isomers of selected compounds

\begin{tabular}{cccc}
\hline Entry & Compounds & $\Delta \mathrm{E}, \mathrm{kcal}^{-\mathrm{mol}^{-1}}(Z-E)$ & d.r. \\
\hline 1 & $(Z)-\mathbf{1 a} /(E)-\mathbf{1 a}$ & 2.88 & $73: 26$ \\
2 & $(Z)-\mathbf{1 b} /(E)-\mathbf{1 b}$ & 2.87 & $75: 24$ \\
3 & $(Z)-\mathbf{1 c} /(E)-\mathbf{1 c}$ & 0.92 & $51: 49$ \\
4 & $(Z)-\mathbf{1 k} /(E)-\mathbf{1 k}$ & 6.05 & $100: 0$ \\
5 & $(Z)-\mathbf{1 4} /(E)-\mathbf{1 4}$ & 6.21 & $100: 0$ \\
\hline
\end{tabular}

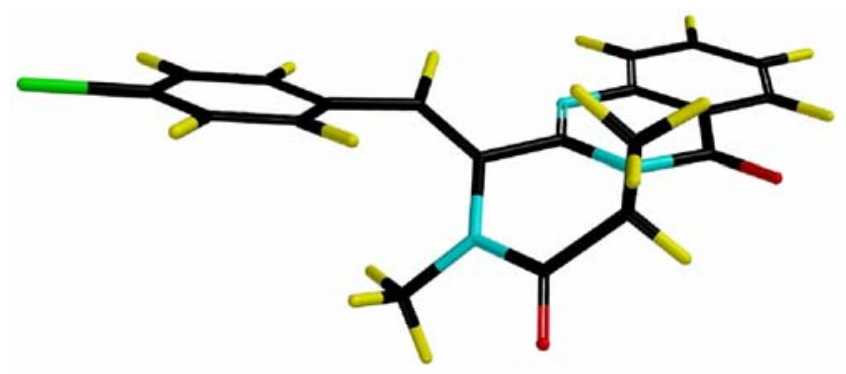

$(Z)-1 \mathrm{a}$

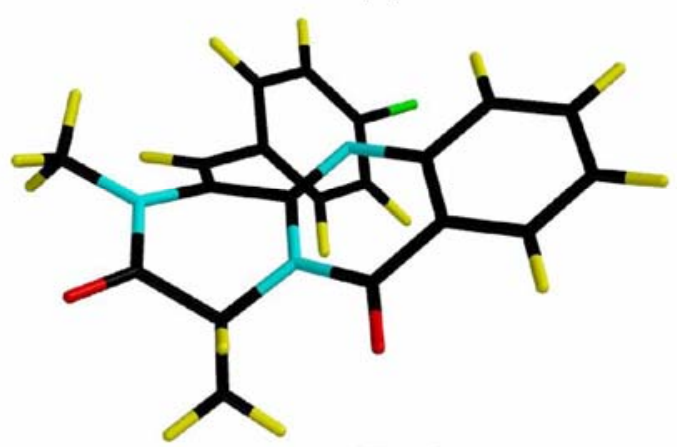

$(E)-1 \mathbf{b}$

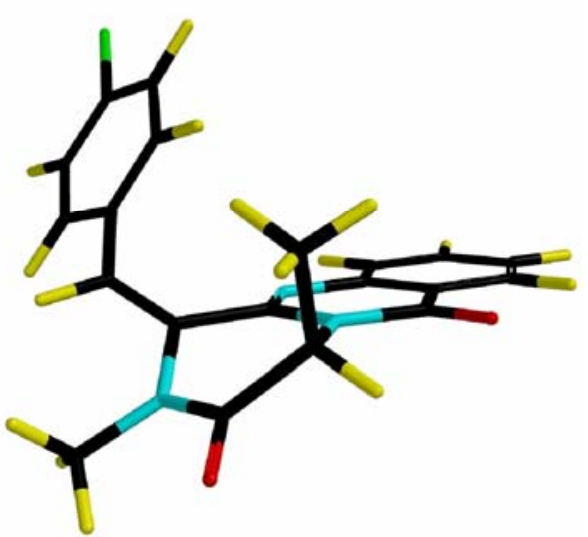

$(E)-\mathbf{1 a}$

\section{Figure 2}

With compounds 1 in hand, we studied their catalytic hydrogenation, which afforded the cis 1,4-disubstituted compounds $\mathbf{3}$ in a fully diastereoselective fashion. Since the $(Z)$ and $(E)$ isomers of compound 1a, not unexpectedly, gave very similar results, we subsequently limited our study to the major $Z$ isomers (Scheme 8, Table 4). In spite of the mild conditions employed, compounds 3 were accompanied by varying amounts of side products 15, arising from the reduction of two bonds of the benzene ring, which were the sole products when prolonged reaction times were used (see the reduction of $\mathbf{1 j}$ ). This behavior may be explained by assuming that the strong conjugation between the amidine and carbonyl groups through the intermediate double bond diminishes the aromaticity of the benzene ring, allowing its partial reduction. ${ }^{23}$ 


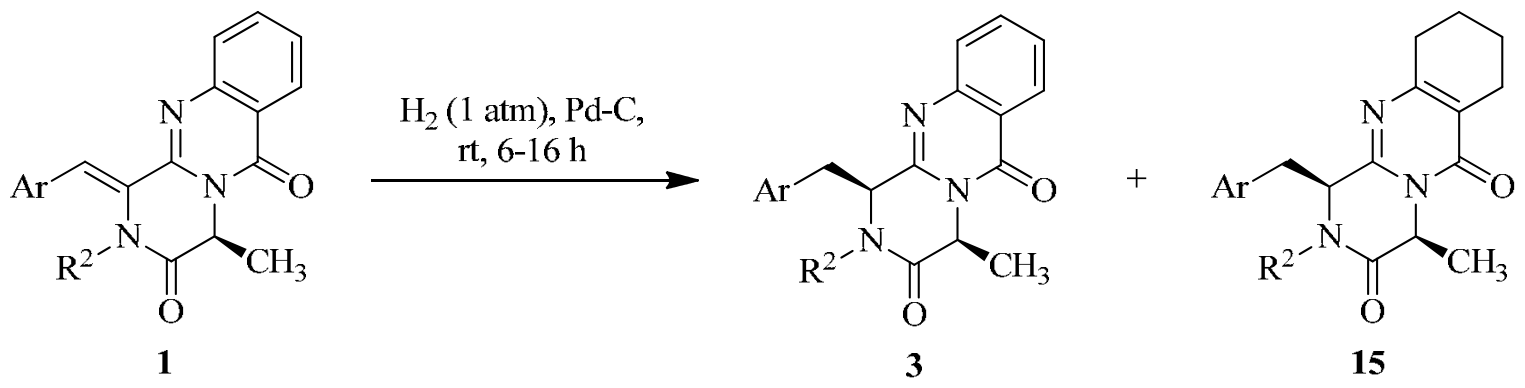

\section{Scheme 8}

Table 4. Scope and yields of the catalytic hydrogenation of compounds $\mathbf{1}$

\begin{tabular}{|c|c|c|c|c|c|}
\hline $\begin{array}{l}\text { Starting } \\
\text { material }\end{array}$ & $\mathrm{Ar}$ & $\mathrm{R}^{2}$ & Time, h & Yield of 3, \% & Yield of 15, \% \\
\hline$(Z)-1 a$ & $4-\mathrm{ClC}_{6} \mathrm{H}_{4}$ & $\mathrm{CH}_{3}$ & 6 & 63 & 17 \\
\hline$(E)-\mathbf{1 a}$ & $4-\mathrm{ClC}_{6} \mathrm{H}_{4}$ & $\mathrm{CH}_{3}$ & 6 & 57 & 15 \\
\hline$(Z)-1 c$ & $4-\mathrm{ClC}_{6} \mathrm{H}_{4}$ & $\mathrm{CH}_{2} \mathrm{CH}_{2} \mathrm{Ph}$ & 6 & 51 & 44 \\
\hline$(Z)-1 d$ & $4-\mathrm{BrC}_{6} \mathrm{H}_{4}$ & $\mathrm{CH}_{3}$ & 6 & 56 & 0 \\
\hline$(Z)-\mathbf{1 f}$ & $3,4-\mathrm{Cl}_{2} \mathrm{C}_{6} \mathrm{H}_{3}$ & $\mathrm{CH}_{3}$ & 6 & 51 & 0 \\
\hline$(Z)-1 g$ & $2,4-\mathrm{F}_{2} \mathrm{C}_{6} \mathrm{H}_{3}$ & $\mathrm{CH}_{3}$ & 6 & $50^{\mathrm{a}}$ & 0 \\
\hline$(Z)-\mathbf{1 h}$ & 1-Benzyl-3-indolyl & $\mathrm{CH}_{3}$ & 16 & 86 & 0 \\
\hline$(Z)-\mathbf{1 i}$ & 1-Tosyl-3-indolyl & $\mathrm{CH}_{3}$ & 6 & 77 & 0 \\
\hline \multirow[t]{2}{*}{$(Z)-\mathbf{1 j}$} & $4-\mathrm{MeOC}_{6} \mathrm{H}_{4}$ & $\mathrm{CH}_{3}$ & 6 & 48 & 30 \\
\hline & & & 16 & 0 & 83 \\
\hline
\end{tabular}

${ }^{\mathrm{a}}$ Based on unrecovered 1g; isolated yield was 30\%.

Interestingly, the diastereoselectivity of the reaction was lost when the $N$-unsubstituted substrate 1k was employed, as shown in Scheme 9. This observation is in sharp contrast with the results described in the literature for similar hydrogenations of arylmethylene-2,5piperazinediones, which give cis products exclusively. To discard any operator- or equipmentassociated bias, we carried out the catalytic hydrogenation of the known compound $17^{12 \mathrm{a}}$ and found that, as expected, it afforded exclusively the cis-disubstituted compound 18.

The loss of diastereoselectivity in the catalytic hydrogenation of $\mathbf{1 k}$ in comparison with that of 17 can be explained by assuming that both the $\alpha$ and $\beta$ faces of the additional aromatic ring interact with the palladium catalyst, partially compensating for the steric hindrance due to the $\mathrm{C}_{4}{ }^{-}$ alkyl substituent. As shown in Figure 3, in the case of $N$-substituted compounds $1 \mathbf{a}-\mathbf{j}$, steric compression between the arylmethylene and $N$-alkyl groups causes a deviation of these substituents from planarity (see also Figure 2), contributing to the blockade of the $\beta$ face and overriding the effect of the bonding interaction due to the aromatic ring. 


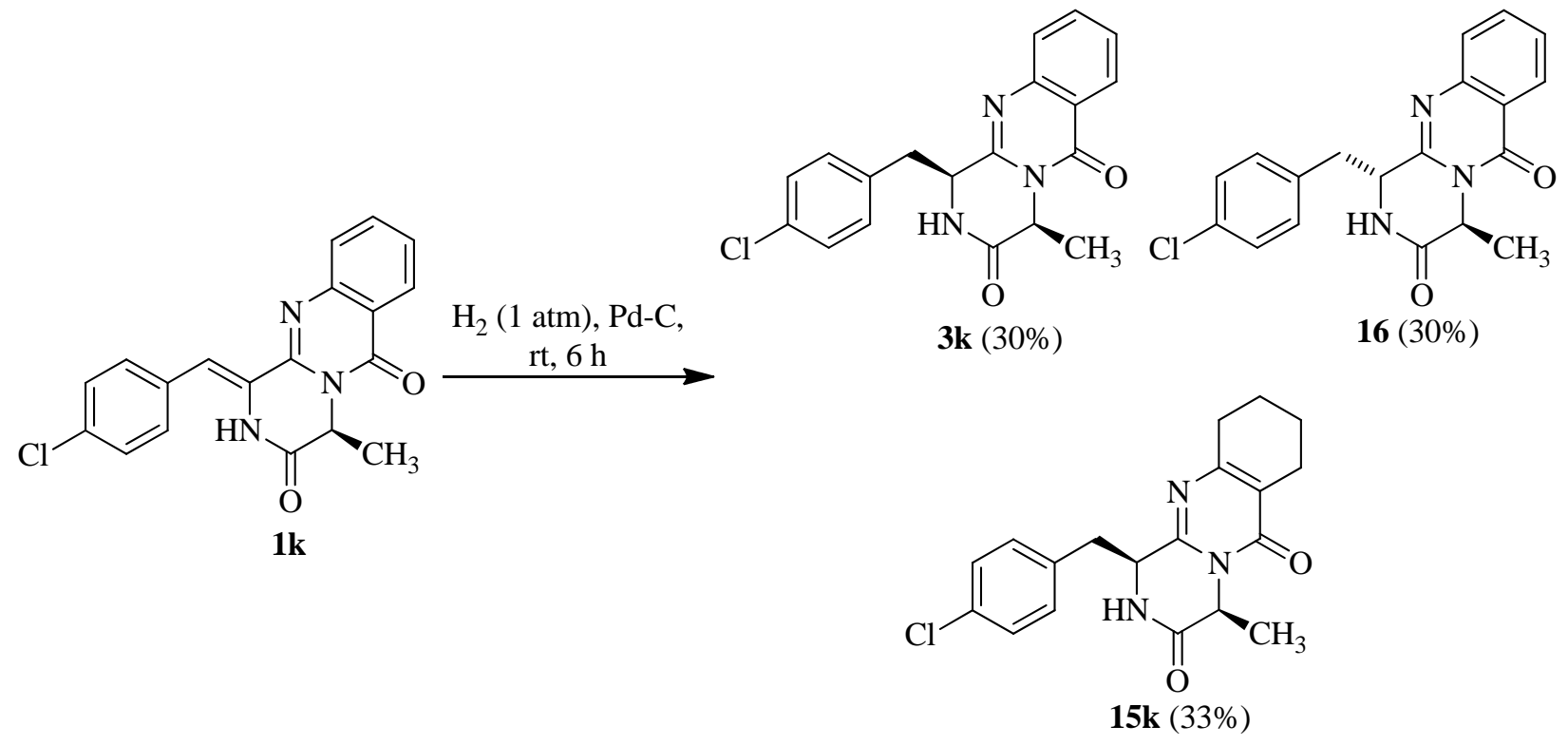<smiles>COc1ccc(/C=C2\NC(=O)[C@@H](C)NC2=O)cc1</smiles>

\section{Scheme 9}

(a)

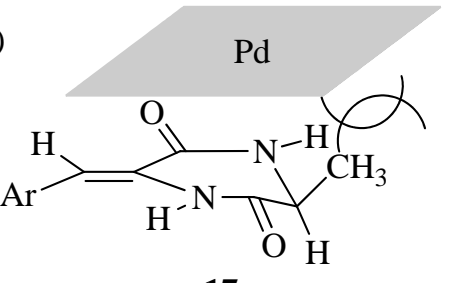

17

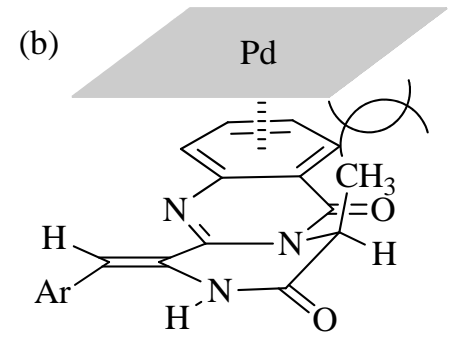

$1 k$ (c)

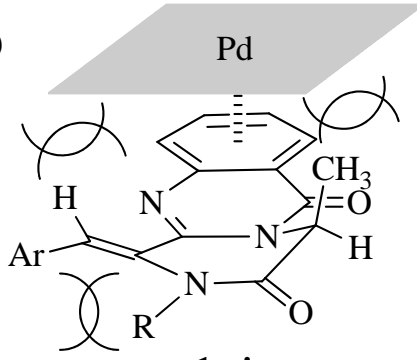

1a-j

Figure 3 


\section{Conclusions}

The direct aldol condensation of pyrazino[2,1-b]quinazoline-3,6-diones, followed or not by catalytic hydrogenation of the exocyclic double bond, provides a very concise route to secoardeemin analogues. These reactions show some significant differences with the ones starting from piperazinedione derivatives, particularly in terms of diastereoselectivity and potential for racemization of carbonyl-adjacent stereocenters. In some cases, the catalytic hydrogenation of 1arylmethylenepyrazino[2,1-b]quinazoline-2,6-diones was accompanied by the unexpected reduction of two double bonds of the benzene ring.

\section{Experimental Section}

General. All reagents (Panreac, Probus, Scharlau, Merck, Fluka, Aldrich) and solvents (SDS) were of commercial quality and were used as received. The expression petroleum ether refers to the $40-60{ }^{\circ} \mathrm{C}$ bp fraction. Reactions were monitored by thin layer chromatography, on aluminium plates coated with silica gel with fluorescent indicator (Scharlau Cf 530, Macherey-Nagel Alugram Sil G/UV 254 ). Separations by flash chromatography were performed on silica gel (SDS 60 ACC 40-63 mm, Scharlau Ge 048). Melting points were measured on a Reichert 723 hot stage microscope, and are uncorrected. Optical rotations were determined in a Perkin-Elmer 240 polarimeter. Infrared spectra were recorded on a Perkin Elmer Paragon 1000 FT-IR spectrophotometer, with all compounds examined as thin films on $\mathrm{NaCl}$ disks (oils) or $\mathrm{KBr}$ pellets (solids). NMR spectra were obtained on Bruker Avance 250 spectrometer operating at $250 \mathrm{MHz}$ for ${ }^{1} \mathrm{H}$ and $63 \mathrm{MHz}$ for ${ }^{13} \mathrm{C}$ (CAI de Resonancia Magnética Nuclear, Universidad Complutense). Elemental analyses were determined by the CAI de Microanálisis Elemental, Universidad Complutense, using a Leco 932 CHNS combustion microanalyzer. HPLC analyses were carried out with a Constametric ${ }^{\circledR} 4100$ instrument fitted with a UV/Visible detector and a Chiralcel-OD (Daicel Chemical Ind., LTD, 0.46 x 25 cm) column.

(4S)-2,4-Dihydro-2,4-dimethyl-1H-pyrazino[2,1-b]quinazoline-3,6-dione (2a). To (3S)-1,3dimethyl-2,5-piperazinedione ${ }^{20}(1 \mathrm{~g}, 7.040 \mathrm{mmol})$, dissolved in dry THF $(50 \mathrm{~mL})$, was added a $0.5 \mathrm{M}$ solution of potassium hexamethyldisilazide in dry toluene $(21 \mathrm{~mL}, 10.5 \mathrm{mmol})$ at $-78{ }^{\circ} \mathrm{C}$ and under an argon atmosphere. After stirring the mixture for $15 \mathrm{~min}$ at the same temperature, a solution of 2-azidobenzoyl chloride (obtained as previously described ${ }^{12 a}$ from $4.5 \mathrm{~g}$ (24.45 mmol) of 2-azidobenzoic acid and $18 \mathrm{~mL}$ of thionyl chloride) in dry THF (50 mL) was added and the reaction mixture was stirred at room temperature for $16 \mathrm{~h}$, and then evaporated. The residue was chromatographed on silica gel, eluting with a 6:1 petroleum ether-ethyl acetate mixture, to yield $2.010 \mathrm{~g}$ (99\%) of (6S)-1-(2-azidobenzoyl)-4,6-dimethylpiperazine-2,5-dione. A solution of this compound (1.039 g, $3.607 \mathrm{mmol})$ and tributylphosphine $(0.914 \mathrm{~mL}, 3.692 \mathrm{mmol})$ in dry toluene $(25 \mathrm{~mL})$ was stirred at $\mathrm{rt}$ for $3 \mathrm{~h}$ under an argon atmosphere. The reaction was then 
evaporated under reduced pressure. The residue was purified by fast chromatography on silica gel, eluting with a 2:1 petroleum ether-dichloromethane mixture, yielding $0.587 \mathrm{~g}$ (71\%) of 2a, as a white solid, with spectral data identical to the ones found in the literature. ${ }^{13}$ (6S)-1-(2Azidobenzoyl)-4,6-dimethylpiperazine-2,5-dione. Pale brown solid; mp $115-117{ }^{\circ} \mathrm{C} ;[\alpha]_{\mathrm{D}}{ }^{25}=-$ 60.0 (c $\left.0.015, \mathrm{CHCl}_{3}\right)$; IR (KBr) v: $2130.0\left(\mathrm{~N}_{3}\right), 1730.0$ and $1674.6(\mathrm{CO}) \mathrm{cm}^{-1}$; ${ }^{1} \mathrm{H}-\mathrm{NMR}$ $\left(\mathrm{CDCl}_{3}\right) \delta 7.47$ (t, $1 \mathrm{H}, J=7.5 \mathrm{~Hz}, \mathrm{H}-5$ '), $7.36(\mathrm{~d}, 1 \mathrm{H}, J=7.5 \mathrm{~Hz}, \mathrm{H}-6$ '), 7.23-7.12 (m, 2H, H3',4'), 5.06 (q, 1H, $J=7.2 \mathrm{~Hz}, \mathrm{H}-6$ ), 4.20 (1d, $1 \mathrm{H}, J=18.4 \mathrm{~Hz}, \mathrm{H}-3$ ), 3.95 (d, $1 \mathrm{H}, J=18.4 \mathrm{~Hz}, \mathrm{H}-$ 3), 3.04 (s, 3H, NCH 3$), 1.58$ (d, 3H, $\left.J=7.2 \mathrm{~Hz}, \mathrm{C}_{6}-\mathrm{CH}_{3}\right) .{ }^{13} \mathrm{C}-\mathrm{NMR}\left(\mathrm{CDCl}_{3}\right) \delta 167.5(\mathrm{C}-2)$, 167.3 (C-5), 165.9 ( $\left.\mathrm{CO}_{\alpha}\right), 136.5$ (C-1'), 136.5 (C-2'), 131.7 (C-4'), 128.1 (C-6'), 125.0 (C-5'), 118.1 (C-3'), 56.5 (C-6), 53.8 (C-3), $33.6\left(\mathrm{NCH}_{3}\right), 18.5\left(\mathrm{C}_{3}-\mathrm{CH}_{3}\right)$. Anal. Calc. for $\mathrm{C}_{13} \mathrm{H}_{13} \mathrm{~N}_{5} \mathrm{O}_{3}$ : C, 54.35; H, 4.56; N, 24.38. Found: C, 54.27; H, 4.87; N, 24.14.

$(4 S, 1 Z)$ - and (4S,1E)-1-(4-Chlorobenzylidene)-2,4-dihydro-2,4-dimethyl-1H-pyrazino[2,1b]quinazoline-3,6-dione, (Z)-1a and (E)-1a. To a solution of 2a (0.30 g, $1.234 \mathrm{mmol})$ and 4chlorobenzaldehyde $(0.26 \mathrm{~g}, 1.851 \mathrm{mmol})$ in dry DMF $(3 \mathrm{~mL})$ at $0{ }^{\circ} \mathrm{C}$ and under an argon atmosphere, a $1 \mathrm{M} \mathrm{KO}^{t} \mathrm{Bu} /{ }^{t} \mathrm{BuOH}$ solution $(1.3 \mathrm{~mL}, 1.3 \mathrm{mmol})$ was added dropwise. The mixture was stirred at rt for $16 \mathrm{~h}$. Then, the reaction was neutralized with acetic acid, poured onto ice and extracted with ethyl acetate. The combined organic extracts were dried (anhydrous $\mathrm{Na}_{2} \mathrm{SO}_{4}$ ) and evaporated. The residue was purified by fast chromatography on silica gel, eluting with a 1:5 ethyl acetate-petroleum ether mixture, yielding $0.331 \mathrm{~g}$ (73\%) of (Z)-1a and $0.130 \mathrm{~g}$ (26\%) of (E)-1a.

Data for (Z)-1a. Yellow solid; mp 202-204 ${ }^{\circ} \mathrm{C}$; IR (KBr) $\vee 1688.2(\mathrm{CO}) \mathrm{cm}^{-1} ;{ }^{1} \mathrm{H}-\mathrm{NMR}\left(\mathrm{CDCl}_{3}\right)$ $\delta 8.28$ (d, $1 \mathrm{H}, J=8.1 \mathrm{~Hz}, \mathrm{H}-7$ ), 7.82-7.70 (m, 2H, H-9,10), 7.50 (t, 1H, $J=8.1 \mathrm{~Hz}, \mathrm{H}-8$ ), 7.45 (d,

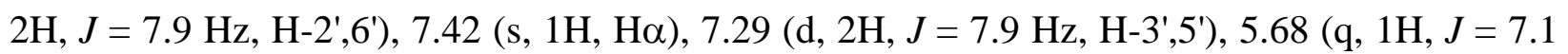
$\mathrm{Hz}, \mathrm{H}-4), 2.97$ (s, 3H, NCH 3$), 1.63$ (d, 3H, $\left.J=7.1 \mathrm{~Hz}, \mathrm{C}_{4}-\mathrm{CH}_{3}\right) ;{ }^{13} \mathrm{C}-\mathrm{NMR}\left(\mathrm{CDCl}_{3}\right) \delta 167.9(\mathrm{C}-$ 3), 159.9 (C-6), 151.4 (C-11a), 147.6 (C-10a), 134.2 (C-4'), 131.1 (C-9), 130.9 (C-1'), 129.8 (C2',6'), 128.3 (C-3',5'), 127.9 (C-7), 127.5 (C-8), 126.7 (C-10), 126.3 (C-1), 119.6 (C $\alpha$ ), 120.4 (C6a), 52.1 (C-4), $35.2\left(\mathrm{NCH}_{3}\right), 17.8\left(\mathrm{C}_{4}-\mathrm{CH}_{3}\right)$. Anal. Calc. for $\mathrm{C}_{20} \mathrm{H}_{16} \mathrm{ClN}_{3} \mathrm{O}_{2}$ : C, 65.67; H, 4.41; N, 11.49. Found: C, 65.55; H, 4.40; N, 11.45.

Data for $(\boldsymbol{E})-\mathbf{1}^{\mathrm{a}}$. Yellow solid; mp 137-139 ${ }^{\circ} \mathrm{C}$; IR $(\mathrm{KBr}) \vee 1682.7(\mathrm{CO}) \mathrm{cm}^{-1} ;{ }^{1} \mathrm{H}-\mathrm{NMR}\left(\mathrm{CDCl}_{3}\right)$ $\delta 8.27$ (d, $1 \mathrm{H}, J=8.0 \mathrm{~Hz}, \mathrm{H}-7$ ), 7.52-7.22 (m, 5H, H-10,2',3',5',6'), 7.58 (t, $1 \mathrm{H}, J=8.0 \mathrm{~Hz}, \mathrm{H}-8$ ), 7.69 (t, $1 \mathrm{H}, J=7.9 \mathrm{~Hz}, \mathrm{H}-9$ ), 6.65 (s, $1 \mathrm{H}, \mathrm{H \alpha}$ ), 5.62 (q, 1H, $J=7.1 \mathrm{~Hz}, \mathrm{H}-4), 3.40$ (s, 3H, $\mathrm{NCH}_{3}$ ), 1.65 (d, 3H, $\left.J=7.1 \mathrm{~Hz}, \mathrm{C}_{4}-\mathrm{CH}_{3}\right) ;{ }^{13} \mathrm{C}-\mathrm{NMR}\left(\mathrm{CDCl}_{3}\right) \delta: 166.8$ (C-3), 159.8 (C-6), 146.8 (C-11a),144.9 (C-10a), 134.1 (C-4'), 134.9 (C-9), 132.6 (C-1'), 131.2 (C-2',6'), 129.1 (C-1), 128.3 (C-3',5'), 128.0 (C-7), 127.8 (C-8), 126.9 (C-10), 121.6 (C $\alpha$ ), 120.7 (C-6a), 51.8 (C-4), $31.8\left(\mathrm{NCH}_{3}\right), 18.3\left(\mathrm{C}_{4}-\mathrm{CH}_{3}\right)$. Anal. Calc. for $\mathrm{C}_{20} \mathrm{H}_{16} \mathrm{ClN}_{3} \mathrm{O}_{2}$ : C, 65.67; H, 4.41; N, 11.49. Found: C, 65.73; H, 4.42; N, 11.44 .

$(4 R, 1 Z)$ - and $(4 R, 1 E)-1-(4-C h l o r o b e n z y l i d e n e)-2,4-d i h y d r o-2,4-d i m e t h y l-1 H$-pyrazino[2,1b]quinazoline-3,6-dione, $(Z)-1 b$ and $(E)-1 b$. Obtained as described for 1a, from compound $\mathbf{2 b}$ (0.20 g, $0.823 \mathrm{mmol}$ ), 4-chlorobenzaldehyde (0.173 g, $1.234 \mathrm{mmol})$, dry DMF (2 mL) and a $1 \mathrm{M}$ $\mathrm{KO}^{\mathrm{t}} \mathrm{Bu} /{ }^{\mathrm{t}} \mathrm{BuOH}$ solution $(0.9 \mathrm{~mL}, 0.9 \mathrm{mmol})$. 
Data for (Z)-1b. Yield $0.225 \mathrm{~g}, 75 \%$; yellow solid (after chromatography eluting with 1:8 EtOAc/petroleum ether); mp 203-205 ${ }^{\circ} \mathrm{C}$. The spectral data are identical to those of compound (Z)-1a. Anal. Calc. for $\mathrm{C}_{20} \mathrm{H}_{16} \mathrm{ClN}_{3} \mathrm{O}_{2}$ : C, 65.67; H, 4.41; N, 11.49. Found: C, 65.63; H, 4.40; N, 11.46 .

Data for $(\boldsymbol{E})$-1b. Yield $0.048 \mathrm{~g}, 24 \%$; yellow solid (after chromatography eluting with 1:8 EtOAc/petroleum ether); mp 136-138 ${ }^{\circ} \mathrm{C}$. The spectral data are identical to those of compound (E)-1a. Anal. Calc. for $\mathrm{C}_{20} \mathrm{H}_{16} \mathrm{ClN}_{3} \mathrm{O}_{2}$ : C, 65.67; H, 4.41; N, 11.49. Found: C, 65.64; H, 4.45; N, 11.44.

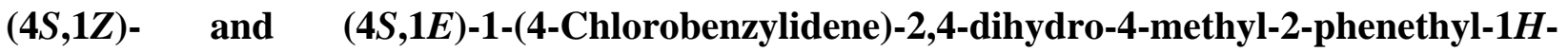
pyrazino[2,1-b]quinazoline-3,6-dione, $(Z)-1 c$ and $(E)-1 c$. Obtained as described for 1a, from compound 2c (0.20 g, $0.60 \mathrm{mmol})$, 4-chlorobenzaldehyde (0.126 g, $0.90 \mathrm{mmol})$, dry DMF (2 $\mathrm{mL})$ and a $1 \mathrm{M} \mathrm{KO}{ }^{\mathrm{t}} \mathrm{Bu} /{ }^{\mathrm{t}} \mathrm{BuOH}$ solution $(0.6 \mathrm{~mL}, 0.6 \mathrm{mmol})$.

Data for (Z)-1c. Yield $0.131 \mathrm{~g}$, 48\%; white crystals (after chromatography eluting with 1:6 EtOAc/petroleum ether); mp 205-207 ${ }^{\circ} \mathrm{C}$; IR (KBr) $v 1687.5$ (CO) $\mathrm{cm}^{-1}$; ${ }^{1} \mathrm{H}-\mathrm{NMR}\left(\mathrm{CDCl}_{3}\right) \delta$ 8.27 (d, 1H, $J=8.0 \mathrm{~Hz}, \mathrm{H}-7$ ), 7.79 (t, 1H, $J=8.0 \mathrm{~Hz}, \mathrm{H}-9$ ), 7.66 (d, 1H, $J=7.9 \mathrm{~Hz}, \mathrm{H}-10$ ), 7.50

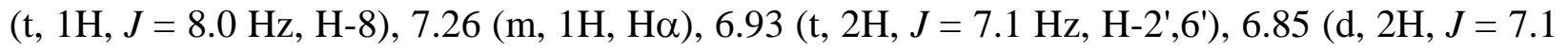
Hz, H-3',5'), 7.38 (s, 5H, Ph), 5.60 (q, 1H, $J=7.2 \mathrm{~Hz}, \mathrm{H}-4$ ), 4.44-4.33 (m, 1H, $\mathrm{CH}_{2} \mathrm{CH}_{2} \mathrm{Ph}$ ), 3.213.05 (m, 1H, $\underline{\mathrm{C}}_{2} \mathrm{CH}_{2} \mathrm{Ph}$ ), 2.81-2.61 (m, 2H, $\left.\mathrm{CH}_{2} \underline{\mathrm{C}}_{2} \mathrm{Ph}\right), 1.54$ (d, $\left.3 \mathrm{H}, J=7.2 \mathrm{~Hz}, \mathrm{C}_{4}-\mathrm{CH}_{3}\right)$; ${ }^{13} \mathrm{C}-$ NMR (CDCl $) \delta 167.9$ (C-3), 159.6 (C-6), 147.6 (C-11a), 147.4 (C-10a), 136.8 (C-1"), 135.1 (C4'), 134.6 (C-9), 131.4 (C-1'), 130.3 (C-2',6'), 130.1 (C-1), 129.1 (C-3',5'), 128.6 (C-2",6"), 128.1 (C-4"), 127.3 (C-3",5"), 127.1 (C-7), 126.8 (C-8), 126.5 (C-10), 121.7 (C-6a), 120.1 (Ca), 51.7 (C-4), $47.5\left(\underline{\mathrm{CH}}_{2} \mathrm{CH}_{2} \mathrm{Ph}\right), 33.1\left(\mathrm{CH}_{2} \underline{\mathrm{CH}_{2}} \mathrm{Ph}\right), 17.1\left(\mathrm{C}_{4}-\underline{\mathrm{CH}_{3}}\right)$; Anal. Calc. for $\mathrm{C}_{27} \mathrm{H}_{22} \mathrm{ClN}_{3} \mathrm{O}_{2}$ : C, 71.13; H, 4.86; N, 9.22. Found: C, 70.89; H, 4.90; N, 9.18.

Data for $(\boldsymbol{E})-1 c$. Yield $0.125 \mathrm{~g}, 46 \%$; white crystals (after chromatography eluting with 1:6 EtOAc/petroleum ether); mp 130-132 ${ }^{\circ} \mathrm{C}$; IR (KBr) v 1683.5 (CO) $\mathrm{cm}^{-1}$; ${ }^{1} \mathrm{H}-\mathrm{NMR}\left(\mathrm{CDCl}_{3}\right)$ $\delta 8.26$ (d, 1H, J = 7.9 Hz, H-7), 7.69 (t, 1H, J = 7.9 Hz, H-9), 7.38-6.87 (m, 10H, H-10,2',3',5',6',

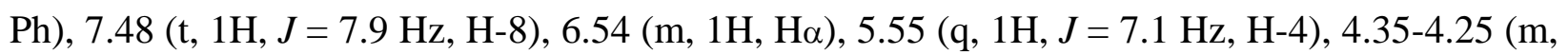
$1 \mathrm{H}, \mathrm{CH}_{2} \mathrm{CH}_{2} \mathrm{Ph}$ ), 4.10-4.00 (m, 1H, $\underline{\mathrm{H}}_{2} \mathrm{CH}_{2} \mathrm{Ph}$ ), 3.15-3.00 (m, 2H, $\mathrm{CH}_{2} \underline{\mathrm{C}}_{2} \mathrm{Ph}$ ), 1.63 (d, 3H, $J=$ $\left.7.1 \mathrm{~Hz}, \mathrm{C}_{4}-\mathrm{CH}_{3}\right)$; ${ }^{13} \mathrm{C}-\mathrm{NMR}\left(\mathrm{CDCl}_{3}\right) \delta 166.4$ (C-3), 159.6 (C-6), 146.7 (C-11a), 144.9 (C-10a), 137.8 (C-1"), 134.7 (C-4'), 133.9 (C-9), 132.2 (C-1'), 130.9 (C-2',6'), 130.2 (C-1), 128.7 (C3',5'), 128.6 (C-2",6"), 128.5 (C-4"), 128.1 (C-3",5"), 127.5 (C-7), 126.8 (C-8), 126.6 (C-10), 122.5 (C-6a), $120.5(\mathrm{C \alpha}), 51.70(\mathrm{C}-4), 46.7\left(\underline{\mathrm{CH}}_{2} \mathrm{CH}_{2} \mathrm{Ph}\right), 33.1\left(\mathrm{CH}_{2} \underline{\mathrm{CH}_{2}} \mathrm{Ph}\right), 17.6\left(\mathrm{C}_{4}-\underline{\mathrm{CH}}_{3}\right)$. Anal. Calc. for $\mathrm{C}_{27} \mathrm{H}_{22} \mathrm{ClN}_{3} \mathrm{O}_{2}$ : C, 71.13; H, 4.86; N, 9.22. Found: C, 70.98; H, 4.89; N, 9.20.

$(4 S, 1 Z)$ - and (4S,1E)-1-(4-Bromobenzylidene)-2,4-dihydro-2,4-dimethyl-1H-pyrazino[2,1b]quinazoline-3,6-dione, $(Z)-1 d$ and $(E)-1 d$. Obtained as described for 1a, from compound 2a (0.20 g, $0.822 \mathrm{mmol}$ ), 4-bromobenzaldehyde (0.288 g, $1.233 \mathrm{mmol})$, dry DMF (2 mL) and a 1M $\mathrm{KO}{ }^{\mathrm{t}} \mathrm{Bu} /{ }^{\mathrm{t}} \mathrm{BuOH}$ solution $(0.9 \mathrm{~mL}, 0.9 \mathrm{mmol})$.

Data for (Z)-1d. Yield $0.150 \mathrm{~g}, 45 \%$; yellow solid (after chromatography eluting with 1:3 EtOAc/petroleum ether); mp 205-207 ${ }^{\circ} \mathrm{C}$; IR (KBr) v: 1692.7 (CO) $\mathrm{cm}^{-1} ;{ }^{1} \mathrm{H}-\mathrm{NMR}$ (DMSO- $d_{6}$ ) $\delta$ 8.05 (d, 1H, $J=8.1 \mathrm{~Hz}, \mathrm{H}-7$ ), 7.62-7.50 (m, 2H, H-9,10), 7.29 (t, 1H, J = 8.1 Hz, H-8), 7.25 (d, 


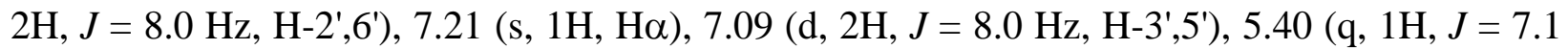
$\mathrm{Hz}, \mathrm{H}-4), 2.78$ (s, 3H, $\mathrm{NCH}_{3}$ ), 1.43 (d, 3H, $J=7.1 \mathrm{~Hz}, \mathrm{C}_{4}-\mathrm{CH}_{3}$ ); ${ }^{13} \mathrm{C}-\mathrm{NMR}\left(\mathrm{DMSO}-d_{6}\right) \delta 168.2$ (C-3), 160.1 (C-6), 147.8 (C-11a), 141.0 (C-10a), 135.2 (C-1'), 132.6 (C-9), 132.6 (C-4'), 132.3 (C-2',6'), 131.1 (C-3',5'), 127.8 (C-7), 127.7 (C-8), 127.3 (C-10), 123.5 (C-1), 121.5 (C-6a), $120.6(\mathrm{C} \alpha), 52.3(\mathrm{C}-4), 35.5\left(\mathrm{NCH}_{3}\right), 17.8\left(\mathrm{C}_{4}-\mathrm{CH}_{3}\right)$. Anal. Calc. for $\mathrm{C}_{20} \mathrm{H}_{16} \mathrm{BrN}_{3} \mathrm{O}_{2}$ : C, 58.55; H, 3.93; N, 10.24. Found: C, 58.32; H, 4.01; N, 10.19.

Data for $(\boldsymbol{E})-1 d$. Yield 0.072 g, 22\%; yellow solid (after chromatography eluting with 1:3 EtOAc/petroleum ether); mp 120-122 ${ }^{\circ} \mathrm{C}$; IR (KBr) v: 1688.5 (CO) cm ${ }^{-1}$; ${ }^{1} \mathrm{H}-\mathrm{NMR}$ (DMSO- $d_{6}$ ) $\delta: 8.08$ (d, 1H, $J=7.6 \mathrm{~Hz}, \mathrm{H}-7), 7.53-7.42$ (m, 2H, H-9,10), 7.35 (t, 1H, J = 7.6 Hz, H-8), 7.25

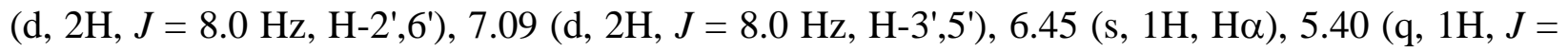
$7.1 \mathrm{~Hz}, \mathrm{H}-4), 3.22$ (s, 3H, $\mathrm{NCH}_{3}$ ), 1.44 (d, 3H, $J=7.1 \mathrm{~Hz}, \mathrm{C}_{4}-\mathrm{CH}_{3}$ ); ${ }^{13} \mathrm{C}-\mathrm{NMR}\left(\mathrm{DMSO}-d_{6}\right) \delta$ : 166.3 (C-3), 159.7 (C-6), 146.7 (C-11a), 144.8 (C-10a), 135.3 (C-1'), 133.1 (C-9), 131.6 (C-4'), 131.3 (C-2',6'), 131.1 (C-3',5'), 127.8 (C-7), 127.7 (C-8), 126.7 (C-10), 122.1 (C-1), 121.5 (C6a), $120.7(\mathrm{C} \alpha), 51.7(\mathrm{C}-4), 31.7\left(\mathrm{NCH}_{3}\right), 18.2\left(\mathrm{C}_{4}-\mathrm{CH}_{3}\right)$. Anal. Calc. for $\mathrm{C}_{20} \mathrm{H}_{16} \mathrm{BrN}_{3} \mathrm{O}_{2}$ : C, 58.55; H, 3.93; N, 10.24. Found: C, 58.58; H, 4.00; N, 10.21.

$(4 S, 1 Z)-$ and $(4 S, 1 E)-2,4-D i h y d r o-2,4-d i m e t h y l-1-(4-n i t r o b e n z y l i d e n e)-1 H$-pyrazino[2,1b]quinazoline-3,6-dione, $(Z)-1 e$ and $(E)-1 e$, and (4S)-2,4-dihydro-2,4-dimethyl-pyrazino[2,1b]quinazoline-1,3,6-trione (4). Obtained as described for 1a, from compound 2a (0.20 g, 0.823 mmol), 4-nitrobenzaldehyde (0.186 g, $1.231 \mathrm{mmol})$, dry DMF (2 mL) and a $1 \mathrm{M} \mathrm{KO}{ }^{\mathrm{t}} \mathrm{Bu} /{ }^{\mathrm{t}} \mathrm{BuOH}$ solution (0.9 mL, $0.9 \mathrm{mmol})$.

Data for (Z)-1e. Yield $0.218 \mathrm{~g}$, 71\%; orange solid (in this case, compound (Z)-1e precipitated when the reaction mixture was poured on ice); mp 295-297 ${ }^{\circ} \mathrm{C}$; IR (KBr) v: 1698.5 (CO) $\mathrm{cm}^{-1}$; ${ }^{1} \mathrm{H}-\mathrm{NMR}\left(\mathrm{DMSO}_{\mathrm{6}}\right.$ ) $\delta 8.30$ (d, 2H, $\left.J=8.1 \mathrm{~Hz}, \mathrm{H}-3^{\prime}, 5^{\prime}\right), 8.17$ (d, $1 \mathrm{H}, J=8.0 \mathrm{~Hz}, \mathrm{H}-7$ ), 7.90 (t, $1 \mathrm{H}, J=8.0 \mathrm{~Hz}, \mathrm{H}-9), 7.79-7.72$ (m, 3H, H-10,2',6'), 7.58 (t, 1H, $J=8.0 \mathrm{~Hz}, \mathrm{H}-8$ ), 7.51 (s, 1H, $\mathrm{H \alpha}$ ), 5.37 (q, 1H, $J=7.1 \mathrm{~Hz}, \mathrm{H}-4), 2.84$ (s, 3H, $\left.\mathrm{NCH}_{3}\right), 1.54$ (d, 3H, $\left.J=7.1 \mathrm{~Hz}, \mathrm{C}_{4}-\mathrm{CH}_{3}\right)$; ${ }^{13} \mathrm{C}-$ NMR (DMSO-d d $\delta 167.3$ (C-3), 166.2 (C-6), 159.3 (C-11a), 147.5 (C-10a), 146.8 (C-4'), 135.1 (C-9), 131.2 (C-1'), 130.9 (C-2',6'), 127.6 (C-7), 127.5 (C-8), 126.6 (C-10), 123.9 (C-3',5'), 122.9 (C-1), 120.6 (C-6a), $118.3(\mathrm{C} \alpha), 51.3(\mathrm{C}-4), 35.7\left(\mathrm{NCH}_{3}\right), 17.8\left(\mathrm{C}_{4}-\mathrm{CH}_{3}\right)$. Anal. Calc. for $\mathrm{C}_{20} \mathrm{H}_{16} \mathrm{~N}_{4} \mathrm{O}_{4}$ : C, 63.82; H, 4.28; N, 14.89. Found: C, 63.91; H, 4.25; N, 14.82.

Data for $(\boldsymbol{E})-1 e$. Yield $0.008 \mathrm{~g}$, 3\%; orange solid (after chromatography eluting with 1:6 EtOAc/petroleum ether); mp 110-112 ${ }^{\circ} \mathrm{C}$; IR (KBr) v: 1687.7 (CO) $\mathrm{cm}^{-1}$; ${ }^{1} \mathrm{H}-\mathrm{NMR}$ (DMSO- $d_{6}$ ) $\delta: 8.27$ (d, 2H, $J=7.8 \mathrm{~Hz}, \mathrm{H}-3$ ',5'), 8.13 (d, 1H, $J=8.1 \mathrm{~Hz}, \mathrm{H}-7$ ), 8.00 (d, 2H, $J=7.8 \mathrm{~Hz}, \mathrm{H}-$ 2',6'), 7.86 (t, 1H, $J=8.1 \mathrm{~Hz}, \mathrm{H}-9$ ), 7.63 (t, 1H, $J=8.1 \mathrm{~Hz}, \mathrm{H}-8), 7.53$ (d, 1H, $J=8.1 \mathrm{~Hz}, \mathrm{H}-10$ ),

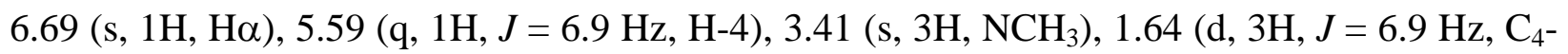

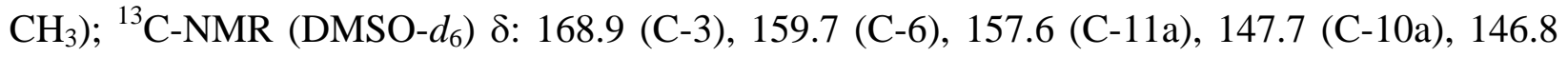
(C-4'), 135.8 (C-9), 131.1 (C-1'), 130.8 (C-2',6'), 130.4 (C-7), 130.2 (C-8), 130.1 (C-10), 127.2 (C-3',5'), 124.3 (C-1), 123.6 (C-6a), 119.5 (Co), 51.9 (C-4), $36.0\left(\mathrm{NCH}_{3}\right), 18.4\left(\mathrm{C}_{4}-\mathrm{CH}_{3}\right)$. Anal. Calc. for $\mathrm{C}_{20} \mathrm{H}_{16} \mathrm{~N}_{4} \mathrm{O}_{4}$ : C, 63.82; H, 4.28; N, 14.89. Found: C, 63.77; H, 4.24; N, 14.86 .

Data for (4). Yield $0.021 \mathrm{~g}, 10 \%$; white solid (after chromatography eluting with 1:6 EtOAc/petroleum ether); mp $166-168{ }^{\circ} \mathrm{C} ;[\alpha]_{\mathrm{D}}{ }^{25}=-3.3\left(\right.$ c $\left.0.3, \mathrm{CHCl}_{3}\right) ;{ }^{24} \mathrm{IR}(\mathrm{KBr})$ v: 1686.0 
(CO) $\mathrm{cm}^{-1}$; ${ }^{1} \mathrm{H}-\mathrm{NMR}\left(\mathrm{CDCl}_{3}\right) \delta 8.32$ (d, $\left.1 \mathrm{H}, J=8.0 \mathrm{~Hz}, \mathrm{H}-7\right), 8.02$ (d, 1H, $\left.J=8.0 \mathrm{~Hz}, \mathrm{H}-10\right)$, 7.89 (t, 1H, $J=8.0 \mathrm{~Hz}, \mathrm{H}-8$ ), 7.64 (t, 1H, $J=8.0 \mathrm{~Hz}, \mathrm{H}-9$ ), 5.60 (q, 1H, $J=7.0 \mathrm{~Hz}, \mathrm{H}-4), 3.42$ (s, $\left.3 \mathrm{H}, \mathrm{NCH}_{3}\right), 1.73$ (d, 3H, $\left.J=7.0 \mathrm{~Hz}, \mathrm{C}_{4}-\mathrm{CH}_{3}\right) ;{ }^{13} \mathrm{C}-\mathrm{NMR}\left(\mathrm{CDCl}_{3}\right) \delta 168.4(\mathrm{C}-3), 159.2(\mathrm{C}-6)$, 158.2 (C-1), 157.1 (C-11a), 146.3 (C-10a), 135.3 (C-9), 129.7 (C-7), 129.6 (C-8), 126.8 (C-10), 121.6 (C-6a), 52.5 (C-4), $27.9\left(\mathrm{NCH}_{3}\right), 21.0\left(\mathrm{C}_{4}-\mathrm{CH}_{3}\right)$. Anal. Calc. for $\mathrm{C}_{13} \mathrm{H}_{11} \mathrm{~N}_{3} \mathrm{O}_{3}$ : C, 60.70; H, 4.31; N, 16.33. Found: C, 60.62; H, 4.36; N, 16.32 .

$(4 S, 1 Z)$ and (4S,1E)-1-(3,4-Dichlorobenzylidene)-2,4-dihydro-2,4-dimethyl-1H-pyrazino[2,1b]quinazoline-3,6-dione, $(Z)-1 f$ and $(E)-1 f$. Obtained as described for 1a, from compound 2a (0.10 g, $0.411 \mathrm{mmol})$, 3,4-dichlorobenzaldehyde (0.108 g, $0.616 \mathrm{mmol})$, dry DMF (1 mL) and a $1 \mathrm{M}$ $\mathrm{KO}^{\mathrm{t}} \mathrm{Bu} /{ }^{\mathrm{t}} \mathrm{BuOH}$ solution $(0.45 \mathrm{~mL}, 0.45 \mathrm{mmol})$.

Data for (Z)-1f. Yield $0.101 \mathrm{~g}, 62 \%$; yellow solid (after chromatography eluting with 1:6 EtOAc/petroleum ether); mp 228-230 ${ }^{\circ} \mathrm{C}$; IR (KBr) v: 1688.7 (CO) $\mathrm{cm}^{-1} ;{ }^{1} \mathrm{H}-\mathrm{NMR}\left(\mathrm{CDCl}_{3}\right) \delta$ 8.27 (d, 1H, $J=7.8 \mathrm{~Hz}, \mathrm{H}-7$ ), 7.81-7.68 (m, 2H, H-9,10), 7.52-7.44 (m, 3H, H-8,2',5'), 7.38 (s, $1 \mathrm{H}, \mathrm{H \alpha}$ ), 7.20 (d, 1H, $J=7.5 \mathrm{~Hz}, \mathrm{H}-6$ ), 5.67 (q, 1H, $J=7.0 \mathrm{~Hz}, \mathrm{H}-4), 2.98$ (s, 3H, NCH $), 1.63$ (d, 3H, $\left.J=7.0 \mathrm{~Hz}, \mathrm{C}_{4}-\mathrm{CH}_{3}\right) ;{ }^{13} \mathrm{C}-\mathrm{NMR}\left(\mathrm{CDCl}_{3}\right) \delta 167.9$ (C-3), 159.8 (C-6), 147.5 (C-11a), 147.2 (C-10a), 136.4 (C-1'), 134.8 (C-3'), 133.3 (C-9), 133.0 (C-4'), 132.9 (C-1), 130.8 (C-5'), 130.5 (C-2'), 128.2 (C-6'), 127.4 (C-7), 127.3 (C-8), 126.8 (C-10), 120.2 (C-6a), 119.3 (C $\alpha$ ), 52.0 (C4), $31.4\left(\mathrm{NCH}_{3}\right), 17.9\left(\mathrm{C}_{4}-\underline{\mathrm{CH}_{3}}\right)$. Anal. Calc. for $\mathrm{C}_{20} \mathrm{H}_{15} \mathrm{Cl}_{2} \mathrm{~N}_{3} \mathrm{O}_{2}$ : C, 60.01; H, 3.78; N, 10.50 . Found: C, 59.87; H, 3.74; N, 10.52.

Data for $(\boldsymbol{E})-1 f$. Yield $0.098 \mathrm{~g}, 23 \%$; yellow solid (after chromatography eluting with 1:6 EtOAc/petroleum ether); mp 179-181 ${ }^{\circ} \mathrm{C}$; IR (KBr) v: 1686.1 (CO) $\mathrm{cm}^{-1} ;{ }^{1} \mathrm{H}-\mathrm{NMR}\left(\mathrm{CDCl}_{3}\right) \delta$ 8.26 (d, 1H, $J=7.9$ Hz, H-7), 7.73-7.64 (m, 2H, H-9,10), 7.48 (t, 1H, $J=7.9$ Hz, H-8), 7.34-7.29

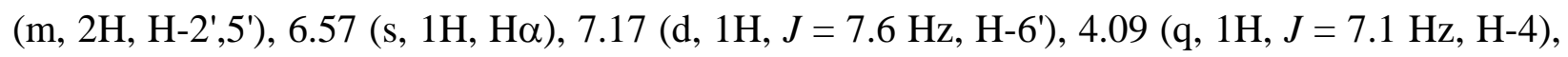
3.39 (s, 3H, $\left.\mathrm{NCH}_{3}\right), 1.62\left(\mathrm{~d}, 3 \mathrm{H}, J=7.1 \mathrm{~Hz}, \mathrm{C}_{4}-\mathrm{CH}_{3}\right) ;{ }^{13} \mathrm{C}-\mathrm{NMR}\left(\mathrm{CDCl}_{3}\right) \delta 166.8(\mathrm{C}-3), 159.7$ (C-6), 146.7 (C-11a), 144.4 (C-10a), 134.8 (C-1'), 134.1 (C-9), 132.3 (C-3'), 131.9 (C-1), 131.8 (C-4'), 131.6 (C-5'), 129.0 (C-2'), 129.6 (C-6'), 127.9 (C-7), 127.6 (C-8), 126.8 (C-10), 120.6 (C6a), $112.0(\mathrm{C} \alpha), 51.7(\mathrm{C}-4), 31.9\left(\mathrm{NCH}_{3}\right), 18.4\left(\mathrm{C}_{4}-\mathrm{CH}_{3}\right)$. Anal. Calc. for $\mathrm{C}_{20} \mathrm{H}_{15} \mathrm{Cl}_{2} \mathrm{~N}_{3} \mathrm{O}_{2}$ : C, 60.01; H, 3.78; N, 10.50. Found: C, 59.84; H, 3.83; N, 10.55.

(4S,1Z)-1-(2,4-Difluorobenzylidene)-2,4-dihydro-2,4-dimethyl-1H-pyrazino[2,1-

b]quinazoline-3,6-dione (1g). Obtained as described for 1a, from compound 2a (0.111 g, 0.451 mmol), 2,4difluorobenzaldehyde $(0.074 \mathrm{~g}, 0.676 \mathrm{mmol})$, dry DMF (1 $\mathrm{mL})$ and a $1 \mathrm{M}$ $\mathrm{KO}^{\mathrm{t}} \mathrm{Bu} /{ }^{\mathrm{t}} \mathrm{BuOH}$ solution $(0.5 \mathrm{~mL}, 0.5 \mathrm{mmol}$ ). Yield $0.144 \mathrm{~g}$, 87\%; white solid (after chromatography eluting with 1:4 EtOAc/petroleum ether); mp 230-232 ${ }^{\circ} \mathrm{C}$; IR (KBr) v: 1691.8 (CO) $\mathrm{cm}^{-1}$; ${ }^{1} \mathrm{H}-\mathrm{NMR}\left(\mathrm{CDCl}_{3}\right) \delta 8.29$ (d, 1H, $\left.J=8.0 \mathrm{~Hz}, \mathrm{H}-7\right), 7.79-7.71$ (m, 2H, H-9,10), 7.49 (t,

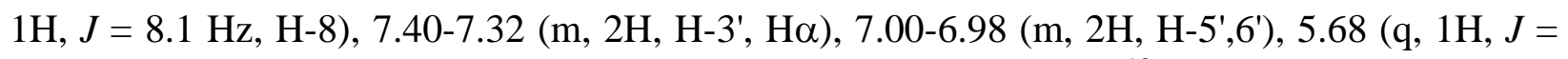
$7.1 \mathrm{~Hz}, \mathrm{H}-4), 2.94$ (s, 3H, NCH$\left.{ }_{3}\right), 1.62$ (d, 3H, $\left.J=7.1 \mathrm{~Hz}, \mathrm{C}_{4}-\mathrm{CH}_{3}\right) ;{ }^{13} \mathrm{C}-\mathrm{NMR}\left(\mathrm{CDCl}_{3}\right) \delta 167.5$ (C-3), 163.4 (dd, $J=253.6$ and $12.3 \mathrm{~Hz}, \mathrm{C}-3$ '), 160.4 (dd, $J=254.2$ and $12.4 \mathrm{~Hz}, \mathrm{C}-4$ '), 159.9 (C6), 158.7 (C-11a), 147.4 (C-10a), 135.0 (C-9), 131.7 (C-1), 131.4 (dd, $J=8.9$ and 8.7 Hz, C-6'), 127.7 (C-7), 127.5 (C-8), 127.0 (C-10), 120.7 (C-6a), 118.1 (dd, $J=18.9$ and $7.9 \mathrm{~Hz}, \mathrm{C}-1^{\prime}$ ), 114.0 (Ca), 112.1 (dd, $J=19.6$ and 5.6 Hz, C-5'), 104.6 (t, $J=25.5$ Hz, C-2'), 52.0 (C-4), 31.5 
$\left(\mathrm{NCH}_{3}\right), 17.6\left(\mathrm{C}_{4}-\underline{C H}_{3}\right)$. Anal. Calc. for $\mathrm{C}_{20} \mathrm{H}_{15} \mathrm{~F}_{2} \mathrm{~N}_{3} \mathrm{O}_{2}$ : C, 65.39; H, 4.12; N, 11.44. Found: C, 65.50; H, 4.15; N, 11.49 .

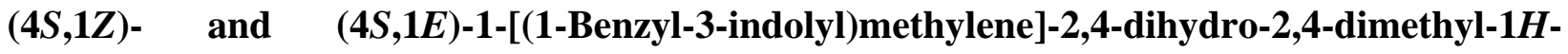
pyrazino[2,1-b]quinazoline-3,6-dione, $(Z)-1 h$ and $(E)-1 h$. Obtained as described for 1a, from compound 2a (0.30 g, $1.234 \mathrm{mmol}$ ), 1-benzylindol-3-carbaldehyde (0.435 g, $1.851 \mathrm{mmol})$, dry DMF (3 mL) and a $1 \mathrm{M} \mathrm{KO}^{\mathrm{t}} \mathrm{Bu} /{ }^{\mathrm{t}} \mathrm{BuOH}$ solution $(1.3 \mathrm{~mL}, 1.3 \mathrm{mmol})$.

Data for (Z)-1h. Yield $0.300 \mathrm{~g}, 53 \%$ as a pale yellow solid (after chromatography eluting with 1:8 EtOAc/petroleum ether); mp 108-110 ${ }^{\circ} \mathrm{C}$; IR (KBr) v: 1680.7 (CO) cm ${ }^{-1}$; ${ }^{1} \mathrm{H}-\mathrm{NMR}$ (DMSO$\left.d_{6}\right) \delta 8.27(\mathrm{~d}, 1 \mathrm{H}, J=8.1 \mathrm{~Hz}, \mathrm{H}-7), 7.84-7.76$ (m, 3H, H-9,10,7'), 7.49 (t, 1H, $J=8.1 \mathrm{~Hz}, \mathrm{H}-8$ ),

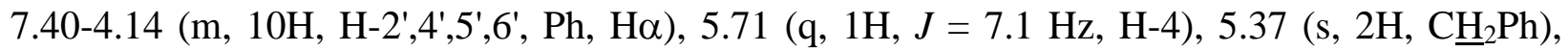
3.10 (s, 3H, $\mathrm{NCH}_{3}$ ), 1.63 (d, 3H, $J=7.1 \mathrm{~Hz}, \mathrm{C}_{4}-\mathrm{CH}_{3}$ ); ${ }^{13} \mathrm{C}-\mathrm{NMR}$ (DMSO-d $\mathrm{d}_{6}$ ) $\delta 168.1$ (C-3), 160.0 (C-6), 151.0 (C-11a), 147.1 (C-10a), 136.3 (C-7a'), 134.6 (C-9), 131.7, 130.2, 128.0, 126.9 (Ph), 129.0 (C-2'), 127.6 (C-1), 127.6 (C-3a'), 127.2 (C-7), 126.8 (C-8), 126.7 (C-10), 126.6 (C4'), 123.1 (C-5'), 121.3 (C-6'), 120.7 (C-6a), 119.7 (C $\alpha$ ), 110.3 (C-7'), 110.2 (C-3'), 52.0 (C-4), $50.5\left(\underline{\mathrm{CH}}_{2} \mathrm{Ph}\right), 33.8\left(\mathrm{NCH}_{3}\right), 17.1\left(\mathrm{C}_{4}-\underline{\mathrm{CH}}_{3}\right)$. Anal. Calc. for $\mathrm{C}_{29} \mathrm{H}_{24} \mathrm{~N}_{4} \mathrm{O}_{2}: \mathrm{C}, 75.63 ; \mathrm{H}, 5.25 ; \mathrm{N}$, 12.17. Found: C, 75.56; H, 5.28; N, 12.13 .

Compound $(\boldsymbol{E})-\mathbf{1 h}$ could not be isolated and its spectral data were obtained from the isomer mixture: ${ }^{1} \mathrm{H}-\mathrm{NMR}$ (DMSO-d $\left.{ }_{6}\right) \delta 8.55$ (s, $1 \mathrm{H}, \mathrm{H}-2$ '), 8.28 (d, $1 \mathrm{H}, J=7.9 \mathrm{~Hz}, \mathrm{H}-7$ ), 7.77-7.12 (m,

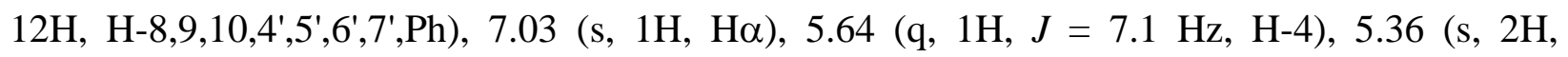
$\left.\mathrm{C}_{2} \mathrm{Ph}\right), 3.50$ (s, 3H, $\left.\mathrm{NCH}_{3}\right), 1.55$ (d, 3H, $J=7.1 \mathrm{~Hz}, \mathrm{C}_{4}-\mathrm{CH}_{3}$ ).

$(4 S, 1 Z)$ and (4S,1E)-2,4-Dihydro-2,4-dimethyl-1-[(1-tosyl-3-indolyl)methylene]-1Hpyrazino[2,1-b]quinazoline-3,6-dione, $(Z)-1 i$ and $(E)-1 i$. Obtained as described for 1a, from compound 2a (0.30 g, $1.234 \mathrm{mmol})$, 1-tosylindol-3-carbaldehyde (0.551 g, $1.851 \mathrm{mmol})$, dry DMF (3 mL) and a $1 \mathrm{M} \mathrm{KO}^{\mathrm{t}} \mathrm{Bu} /{ }^{\mathrm{t}} \mathrm{BuOH}$ solution (1.3 mL, $\left.1.3 \mathrm{mmol}\right)$.

Data for (Z)-1i. Yield $0.227 \mathrm{~g}$, 36\%; red solid (after chromatography eluting with 1:8 EtOAc/petroleum ether); mp 200-202 ${ }^{\circ} \mathrm{C}$; IR (KBr) $\vee 1689.4$ (CO) $\mathrm{cm}^{-1} ;{ }^{1} \mathrm{H}-\mathrm{NMR}\left(\mathrm{CDCl}_{3}\right) \delta$ 8.09 (d, 1H, $J=8.0 \mathrm{~Hz}, \mathrm{H}-7$ ), 7.83 (d, 1H, $J=8.0$ Hz, H-7'), 7.66-7.53 (m, 2H, H-9,10), 7.61 (d,

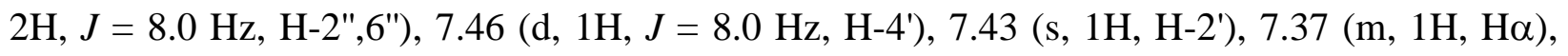
7.31 (t, 1H, $J=8.0 \mathrm{~Hz}, \mathrm{H}-8$ ), 7.13 (t, 1H, $J=8.0 \mathrm{~Hz}, \mathrm{H}-5$ '), 7.21 (t, $1 \mathrm{H}, J=8.0 \mathrm{~Hz}, \mathrm{H}-6$ '), 7.05 (d, 2H, $J=8.0 \mathrm{~Hz}, \mathrm{H}-3 ", 5 "), 5.50$ (q, 1H, $J=7.0 \mathrm{~Hz}, \mathrm{H}-4$ ), 2.74 (s, 3H, NCH ), 2.14 (s, 3H, $\left.\mathrm{C}_{6} \mathrm{H}_{4} \underline{\mathrm{C}}_{3}\right), 1.49$ (d, 3H, $\left.J=7.0 \mathrm{~Hz}, \mathrm{C}_{4}-\mathrm{CH}_{3}\right) ;{ }^{13} \mathrm{C}-\mathrm{NMR}\left(\mathrm{CDCl}_{3}\right) \delta 168.1$ (C-3), 160.3 (C-6), 148.0 (C-11a), 146.1 (C-10a), 135.3 (C-9), 135.1 (C-7'a,4”), 132.1 (C-1"), 130.5 (C-2",6"), 129.9 (C-1), 129.5 (C-2'), 127.9 (C-3'a), 127.6 (C-7), 127.3 (C-3",5"), 126.7 (C-8), 126.1 (C-10), 124.6 (C-4',5'), 120.6 (C-6a), 120.4 (C-6'), 116.0 (C-3'), 114.2 (C-7'), 113.4 (Co), 52.4 (C-4), 34.5 $\left(\mathrm{NCH}_{3}\right), 22.0\left(\mathrm{C}_{6} \mathrm{H}_{4} \underline{\mathrm{C}} \mathrm{H}_{3}\right), 18.1\left(\mathrm{C}_{4}-\underline{\mathrm{CH}}_{3}\right)$. Anal. Calc. for $\mathrm{C}_{29} \mathrm{H}_{24} \mathrm{~N}_{4} \mathrm{O}_{4} \mathrm{~S}: \mathrm{C}, 66.40 ; \mathrm{H}, 4.61 ; \mathrm{N}$, 10.68: Found: C, 66.31; H, 4.63; N, 10.65.

Data for $(\boldsymbol{E})$-1i. Yield $0.103 \mathrm{~g}, 16 \%$; orange oil (after chromatography eluting with 1:8 EtOAc/petroleum ether); IR (NaCl) v: 1686.4 (CO) $\mathrm{cm}^{-1} ;{ }^{1} \mathrm{H}-\mathrm{NMR}\left(\mathrm{CDCl}_{3}\right) \delta 8.15$ (d, $1 \mathrm{H}, J=$ $7.8 \mathrm{~Hz}, \mathrm{H}-7$ ), 7.82 (d, 1H, $J=7.8 \mathrm{~Hz}, \mathrm{H}-7$ ), 7.70-7.66 (m, 2H, H-9,10), 7.65 (d, 2H, J = 7.5 Hz,

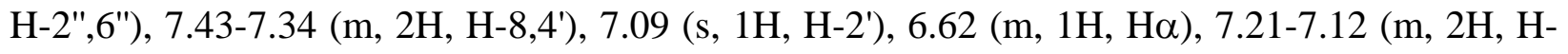


5',6'), 7.02 (d, 2H, $J=7.5$ Hz, H-3",5"), 5.50 (q, 1H, J = 7.1 Hz, H-4), 3.32 (s, 3H, NCH $), 2.18$ (s, 3H, $\left.\mathrm{C}_{6} \mathrm{H}_{4} \mathrm{CH}_{3}\right), 1.39$ (d, 3H, $\left.J=7.1 \mathrm{~Hz}, \mathrm{C}_{4}-\mathrm{CH}_{3}\right) ;{ }^{13} \mathrm{C}-\mathrm{NMR}\left(\mathrm{CDCl}_{3}\right) \delta 167.3(\mathrm{C}-3), 160.1$ (C6), 147.1 (C-11a), 145.7 (C-10a), 135.5 (C-7'a,4”), 134.6 (C-9), 131.6 (C-1"), 131.0 (C-1), 130.4 (C-2",6"), 129.6 (C-2',3'a), 128.3 (C-7), 127.7 (C-8,10), 127.4 (C-3",5"), 125.6 (C-4'), 123.9 (C-

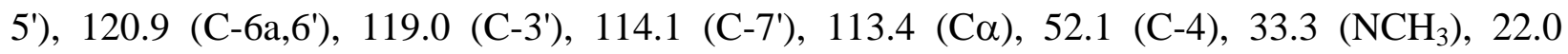
$\left(\mathrm{C}_{6} \mathrm{H}_{4} \underline{\mathrm{CH}_{3}}\right), 18.2\left(\mathrm{C}_{4}-\underline{\mathrm{CH}_{3}}\right)$. Anal. Calc. for $\mathrm{C}_{29} \mathrm{H}_{24} \mathrm{~N}_{4} \mathrm{O}_{4} \mathrm{~S}: \mathrm{C}, 66.40 ; \mathrm{H}, 4.61 ; \mathrm{N}, 10.68$. Found: $\mathrm{C}$, 66.38; H, 4.58; N, 10.69.

Reaction of (2a) with 4-methoxybenzaldehyde. This reaction was carried out as described for the preparation of 1a, from compound $2 \mathbf{a}(0.10 \mathrm{~g}, 0.411 \mathrm{mmol})$, 4-methoxybenzaldehyde (0.074 $\mathrm{mL}, 0.616 \mathrm{mmol})$, dry DMF (1 mL) and a $1 \mathrm{M} \mathrm{KO}^{\mathrm{t}} \mathrm{Bu} /{ }^{\mathrm{t}} \mathrm{BuOH}$ solution $(1.0 \mathrm{~mL}, 1.0 \mathrm{mmol})$, and after chromatography eluting with 1:6 EtOAc/petroleum ether, compounds $(4 S, 1 Z)$ - and $(4 S, 1 E)$ 2,4-dihydro-1-(4-methoxybenzylidene)-2,4-dimethyl-1H-pyrazino[2,1-b]quinazoline-3,6-dione (Z)-1j, $\quad(\boldsymbol{E})-\mathbf{1 j}, \quad(4 S, 1 Z)-\quad$ and $\quad(4 S, 1 E)-2,4-d i h y d r o-1-(4-m e t h o x y b e n z y l i d e n e)-2-[2-(4-$ methoxyphenyl)-2-oxoethyl]-4-methyl-1H-pyrazino[2,1-b]quinazoline-3,6-dione (Z)-5, and (E)$\mathbf{5}$ were obtained. When 1 equivalent of base was used, the reaction only yielded $63 \%$ of $(\mathbf{Z}) \mathbf{- 1} \mathbf{j}$, traces of $(\boldsymbol{E})-\mathbf{1 j}$ together with recovered starting material.

Compound (Z)-1j. Yield 0.093 g, 63\%; yellow solid (after chromatography eluting with 1:6 EtOAc/petroleum ether); mp 173-175 ${ }^{\circ} \mathrm{C}$; IR (KBr) $v 1684.1$ (CO) $\mathrm{cm}^{-1}$; ${ }^{1} \mathrm{H}-\mathrm{NMR}\left(\mathrm{CDCl}_{3}\right) \delta$ 8.26 (d, 1H, $J=8.1 \mathrm{~Hz}, \mathrm{H}-7$ ), 7.79-7.71 (m, 2H, H-9,10), 7.46 (t, 1H, J = 8.1 Hz, H-8), 7.32 (d,

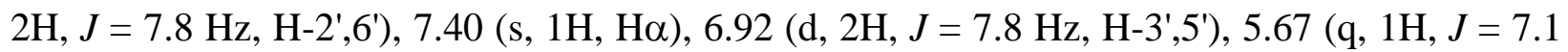
$\mathrm{Hz}, \mathrm{H}-4), 3.82$ (s, 3H, $\mathrm{OCH}_{3}$ ), 3.00 (s, 3H, $\mathrm{NCH}_{3}$ ), 1.61 (d, 3H, $J=7.1 \mathrm{~Hz}, \mathrm{C}_{4}-\mathrm{CH}_{3}$ ); ${ }^{13} \mathrm{C}-\mathrm{NMR}$ $\left(\mathrm{CDCl}_{3}\right) \delta 168.1$ (C-3), 160.2 (C-6), 160.0 (C-4'), 148.2 (C-11a), 147.7 (C-10a), 134.9 (C-9), 131.1 (C-2',6'), 129.6 (C-1'), 127.4 (C-7), 127.1 (C-8), 127.0 (C-10), 125.5 (C-1), 123.0 (C $\alpha)$, 120.3 (C-6a), 114.3 (C-3',5'), $55.5\left(\mathrm{OCH}_{3}\right), 51.1(\mathrm{C}-4), 34.9\left(\mathrm{NCH}_{3}\right), 17.5\left(\mathrm{C}_{4}-\mathrm{CH}_{3}\right)$. Anal. Calc. for $\mathrm{C}_{21} \mathrm{H}_{19} \mathrm{~N}_{3} \mathrm{O}_{3}$ : C, 69.79; H, 5.30; N, 11.63. Found: C, 69.83; H, 5.33; N, 11.60 .

Compound (E)-1j. Yield 0.023g, 16\%; yellow solid (after chromatography eluting with 1:6 EtOAc/petroleum ether); mp 208-210 ${ }^{\circ} \mathrm{C}$; IR (KBr) $v 1684.0$ (CO) $\mathrm{cm}^{-1}$; ${ }^{1} \mathrm{H}-\mathrm{NMR}\left(\mathrm{CDCl}_{3}\right) \delta$ 8.28 (d, 1H, $J=8.0 \mathrm{~Hz}, \mathrm{H}-7$ ), 7.68 (t, 1H, $J=7.9 \mathrm{~Hz}, \mathrm{H}-9$ ), 7.48 (t, $1 \mathrm{H}, J=7.9 \mathrm{~Hz}, \mathrm{H}-8$ ), 7.40 (d, $1 \mathrm{H}, J=8.0 \mathrm{~Hz}, \mathrm{H}-10$ ), 7.39 (d, 2H, $J=7.8 \mathrm{~Hz}, \mathrm{H}-2$ ',6'), 6.79 (d, 2H, $J=7.8 \mathrm{~Hz}, \mathrm{H}-3$ ',5'), 6.66 (s, $1 \mathrm{H}, \mathrm{H \alpha}$ ), 5.62 (q, $1 \mathrm{H}, J=7.1 \mathrm{~Hz}, \mathrm{H}-4), 3.80$ (s, 3H, $\mathrm{OCH}_{3}$ ), 3.39 (s, 3H, $\left.\mathrm{NCH}_{3}\right), 1.64$ (d, 3H, $J=$ $\left.7.1 \mathrm{~Hz}, \mathrm{C}_{4}-\mathrm{CH}_{3}\right) ;{ }^{13} \mathrm{C}-\mathrm{NMR}\left(\mathrm{CDCl}_{3}\right) \delta 167.0$ (C-3), 159.9 (C-6), 158.9 (C-4'), 147.1 (C-11a), 145.8 (C-10a), 134.8 (C-9), 131.7 (C-2',6'), 127.8 (C-7), 127.7 (C-8), 126.9 (C-10), 126.0 (C1',1"), 123.5 (C-6a), 120.8 (C $\alpha), 113.6$ (C-3',5'), $55.4\left(\mathrm{OCH}_{3}\right), 51.9(\mathrm{C}-4), 31.9\left(\mathrm{NCH}_{3}\right), 18.0$ $\left(\mathrm{C}_{4}-\underline{C H}_{3}\right)$. Anal. Calc. for $\mathrm{C}_{21} \mathrm{H}_{19} \mathrm{~N}_{3} \mathrm{O}_{3}$ : C, 69.79; H, 5.30; N, 11.63. Found: C, 69.67; H, 5.34; N, 11.64.

Compound (Z)-5. Yield 0.025 g, 12\%; orange solid (after chromatography eluting with 1:6 EtOAc/petroleum ether); mp 96-98 ${ }^{\circ} \mathrm{C}$; IR (KBr) $\vee 1682.2$ and 1602.8 (CO) $\mathrm{cm}^{-1}$; ${ }^{1} \mathrm{H}-\mathrm{NMR}$ $\left(\mathrm{CDCl}_{3}\right) \delta: 8.30$ (d, 1H, J= $\left.7.9 \mathrm{~Hz}, \mathrm{H}-7\right), 7.80-7.72$ (m, 2H, H-8,9), 7.63 (d, 2H, J = 7.4 Hz, H-

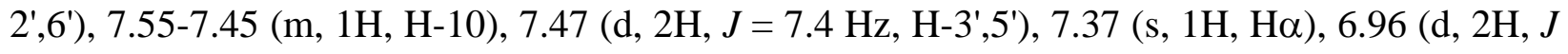
= $7.3 \mathrm{~Hz}, \mathrm{H}-3 ", 5 "), 6.79$ (d, 2H, $J=7.3 \mathrm{~Hz}, \mathrm{H}-2 ", 6 "), 5.77$ (q, 1H, J = $7.1 \mathrm{~Hz}, \mathrm{H}-4$ ), 5.54 (d, 1H, 
$J=17.4 \mathrm{~Hz}, \mathrm{NCH}_{2}$ ), $4.41\left(\mathrm{~d}, 1 \mathrm{H}, J=17.4 \mathrm{~Hz}, \mathrm{NCH}_{2}\right.$ ), 3.87 and 3.83 (s, 6H, $\left.\mathrm{OCH}_{3}\right), 1.67$ (d, 3H, $\left.J=7.1 \mathrm{~Hz}, \mathrm{C}_{4}-\mathrm{CH}_{3}\right) ;{ }^{13} \mathrm{C}-\mathrm{NMR}\left(\mathrm{CDCl}_{3}\right) \delta 189.8\left(\mathrm{COCH}_{2}\right), 169.1$ (C-3), 163.8 (C-6), 160.2 and 160.0 (C-4',4"), 148.2 (C-11a), 147.8 (C-10a), 134.5 (C-9), 130. 9 and 130.1 (C-2',2",6',6"), 127.4 (C-7), 128.9 and 127.9 (C-1',1"), 127.8 (C-8), 126.7 (C-10), 125.1 (C-1), 123.1 (C-6a), $120.3(\mathrm{C} \alpha), 114.3$ and $113.7\left(\mathrm{C}-3^{\prime}, 3^{\prime}, 5 ', 5 "\right), 55.3$ and $55.3\left(2 \mathrm{OCH}_{3}\right), 55.2(\mathrm{C}-4), 55.9\left(\mathrm{COCH}_{2}\right)$, $17.1\left(\mathrm{C}_{4}-\mathrm{CH}_{3}\right)$. Anal. Calc. for $\mathrm{C}_{29} \mathrm{H}_{25} \mathrm{~N}_{3} \mathrm{O}_{5}$ : C, 70.29; H, 5.09; N, 8.48. Found: C, 70.19; H, 5.06; N, 8.42.

Compound (E)-5. Yield 0.016 g, 8\%; orange solid (after chromatography eluting with 1:6 EtOAc/petroleum ether); mp 94-96 ${ }^{\circ} \mathrm{C}$; IR (KBr) v: 1681.7 and 1601.6 (CO) $\mathrm{cm}^{-1}$; ${ }^{1} \mathrm{H}-\mathrm{NMR}$ $\left(\mathrm{CDCl}_{3}\right) \delta 8.29$ (d, 1H, $\left.J=8.0 \mathrm{~Hz}, \mathrm{H}-7\right), 7.99$ (d, 2H, $J=7.3 \mathrm{~Hz}, \mathrm{H}-2$ ',6'), 7.67 (d, 1H, $J=7.9$ Hz, H-9), 7.48 (t, 1H, $J=7.9$ Hz, H-8), 7.39 (d, 1H, $J=7.9$ Hz, H-10), 7.32 (d, 2H, $J=7.3 \mathrm{~Hz}$,

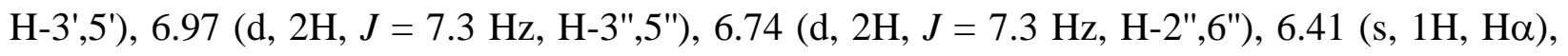
5.68 (q, 1H, $J=7.1 \mathrm{~Hz}, \mathrm{H}-4), 5.55$ (d, $1 \mathrm{H}, J=17.5 \mathrm{~Hz}, \mathrm{NCH}_{2}$ ), 4.87 (d, 1H, $J=17.5 \mathrm{~Hz}, \mathrm{NCH}_{2}$ ), 3.87 and $3.83\left(\mathrm{~s}, 6 \mathrm{H}, \mathrm{OCH}_{3}\right), 1.67$ (d, 3H, $\left.J=7.1 \mathrm{~Hz}, \mathrm{C}_{4}-\mathrm{CH}_{3}\right) ;{ }^{13} \mathrm{C}-\mathrm{NMR}\left(\mathrm{CDCl}_{3}\right) \delta 189.8$ ( $\left.\mathrm{COCH}_{2}\right), 167.1$ (C-3), 164.1 (C-6), 159.8 and 159.6 (C-4',4"), 146.9 (C-11a), 145.6 (C-10a), 134.5 (C-9), 131.4 and 130.5 (C-2',2",6',6"), 127.6 (C-7), 127.5 (C-8), 126.7 (C-10), 128.6 and 127.2 (C-1',1"), 125.6 (C-1), 123.6 (C $\alpha$ ), 120.6 (C-6a), 114.0 and 113.2 (C-3',3",5',5"), 57.5 and $55.1\left(2 \mathrm{OCH}_{3}\right), 51.9(\mathrm{C}-4), 51.7\left(\mathrm{COCH}_{2}\right), 17.5\left(\mathrm{C}_{4}-\underline{C H}_{3}\right)$. Anal. Calc. for $\mathrm{C}_{29} \mathrm{H}_{25} \mathrm{~N}_{3} \mathrm{O}_{5}$ : C, 70.29; H, 5.09; N, 8.48. Found: C, 70.15; H, 5.03; N, 8.51.

(4S,1Z)-1-(4-Chlorobenzylidene)-2,4-dihydro-4-methyl-1H-pyrazino[2,1-b]quinazoline-3,6-

dione (1k). To a solution of (4S)-2-acetyl-1,2,3,4-tetrahydro-4-methyl-5 $\mathrm{H}$-pyrazino[2,1b]quinazoline-3,6-dione ${ }^{3 \mathrm{~b}}(0.148 \mathrm{~g}, 0.546 \mathrm{mmol})$ and 4-chlorobenzaldehyde (0.115 g, 0.819 $\mathrm{mmol})$ in dry DMF $(1 \mathrm{~mL})$ at $0{ }^{\circ} \mathrm{C}$ and under an argon atmosphere, was added dropwise a $1 \mathrm{M}$ $\mathrm{KO}^{t} \mathrm{Bu} /{ }^{\mathrm{t}} \mathrm{BuOH}$ solution $(0.573 \mathrm{~mL}, 0.573 \mathrm{mmol})$. The mixture was stirred at rt for $16 \mathrm{~h}$. Then, the reaction was neutralized with acetic acid, poured onto ice, and the yellow solid that precipitated was collected by filtration and identified as $1 \mathbf{k}$. Yield 0.180 g, 94\%; mp $173-175{ }^{\circ} \mathrm{C}$; $[\alpha]_{\mathrm{D}}{ }^{25}=-471.6\left(c \mathrm{0.03}, \mathrm{CHCl}_{3}\right)$; IR (KBr) $\vee 1687.1(\mathrm{CO}) \mathrm{cm}^{-1} ;{ }^{1} \mathrm{H}-\mathrm{NMR}\left(\mathrm{CDCl}_{3}\right) \delta 8.28(\mathrm{~d}, 1 \mathrm{H}$, $J=8.1 \mathrm{~Hz}, \mathrm{H}-7), 7.82-7.75$ (m, 3H, H-9,10,NH), 7.51 (t, 1H, $J=8.1 \mathrm{~Hz}, \mathrm{H}-8$ ), 7.48-7.38 (m, 4H,

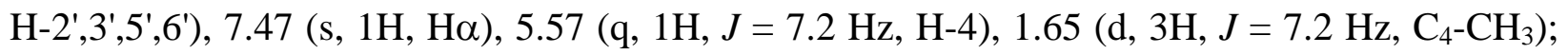
${ }^{13} \mathrm{C}-\mathrm{NMR}\left(\mathrm{CDCl}_{3}\right) \delta 166.1$ (C-3), 160.0 (C-6), 151.0 (C-11a), 146.9 (C-10a), 134.8 (C-9), 134.6 (C-1'), 131.5 (C-4'), 129.8 (C-2',6'), 129.6 (C-3',5'), 127.5 (C-7), 127.3 (C-8), 126.8 (C-10), 126.3 (C-1), 120.2 (C-6a), $114.6(\mathrm{C} \alpha), 51.6(\mathrm{C}-4), 19.3\left(\mathrm{C}_{4}-\mathrm{CH}_{3}\right)$. Anal. Calc. for $\mathrm{C}_{19} \mathrm{H}_{14} \mathrm{ClN}_{3} \mathrm{O}_{2}$ : C, 64.87; H, 4.01; N, 11.94. Found: C, 64.83; H, 4.05; N, 11.90.

(3S)-4-Acetyl-1,3-dimethylpiperazine-2,5-dione (13). A solution of (3S)-1,3dimethylpiperazine-2,5-dione $(0.5 \mathrm{~g}, 3.52 \mathrm{mmol})$ in acetic anhydride $(10 \mathrm{~mL})$ was refluxed at $140{ }^{\circ} \mathrm{C}$ for $7 \mathrm{~h}$ with exclusion of the moisture. The excess of anhydride was evaporated under reduced pressure yielding compound 13 as a yellow oil. Yield 0,636 g, 98\%; $[\alpha]_{\mathrm{D}}{ }^{25}=-24$ (c 0.05, $\mathrm{CHCl}_{3}$ ); IR $(\mathrm{NaCl}) \vee 1709.5$ and $1672.3(\mathrm{CO}) \mathrm{cm}^{-1} ;{ }^{1} \mathrm{H}-\mathrm{NMR}\left(\mathrm{CDCl}_{3}\right) \delta 5.03(\mathrm{q}, 1 \mathrm{H}, J=$ $7.2 \mathrm{~Hz}, \mathrm{H}-3$ ), 4.24 (d, 1H, $J=18.3 \mathrm{~Hz}, \mathrm{H}-6), 3.94$ (d, 1H, $J=18.3 \mathrm{~Hz}, \mathrm{H}-6), 2.98$ (s, 3H, NCH $)$, $2.52\left(\mathrm{~s}, 3 \mathrm{H}, \mathrm{COCH}_{3}\right), 1.43\left(\mathrm{~d}, 3 \mathrm{H}, J=7.2 \mathrm{~Hz}, \mathrm{C}_{3}-\mathrm{CH}_{3}\right) ;{ }^{13} \mathrm{C}-\mathrm{NMR}\left(\mathrm{CDCl}_{3}\right) \delta 171.0\left(\mathrm{~N}_{1} \mathrm{COCH}_{3}\right)$, 
167.6 (C-2), 166.5 (C-5), 52.6 (C-3), 52.9 (C-6), $33.3\left(\mathrm{NCH}_{3}\right), 27.1\left(\mathrm{COCH}_{3}\right), 18.0\left(\mathrm{C}_{3}-\underline{\mathrm{CH}}_{3}\right)$. Anal. Calc. for $\mathrm{C}_{8} \mathrm{H}_{12} \mathrm{~N}_{2} \mathrm{O}_{3}$ : C, 52.17; H, 6.57; N, 15.21. Found: C, 51.94; H, 6.55; N, 15.26. $(6 S, 3 Z)$ - and $(6 S, 3 E)$-4-Acetyl-3-(4-chlorobenzylidene)-4,6-dimethylpiperazine-2,5-dione, $(Z)-14$ and $(E)-14$. Obtained as described for 1a, from compound 13 (0.100 g, 0,550 mmol), 4chlorobenzaldehyde $(0.100 \mathrm{~mL}, 0.714 \mathrm{mmol})$, dry $\mathrm{DMF}(1 \mathrm{~mL})$ and a $1 \mathrm{M} \mathrm{KO}{ }^{\mathrm{t}} \mathrm{Bu} /{ }^{\mathrm{t}} \mathrm{BuOH}$ solution (0.6 mL, $0.6 \mathrm{mmol})$.

Compound (Z)-14. Yield 0.019 g, 11\%; yellow solid (after chromatography eluting with 1:8 $\mathrm{Cl}_{2} \mathrm{CH}_{2}$ /petroleum ether); mp $129-131^{\circ} \mathrm{C} ;[\alpha]_{\mathrm{D}}{ }^{25}=-158,4$ (c 0.25, $\mathrm{CHCl}_{3}$ ); IR (KBr) v: 1703.6

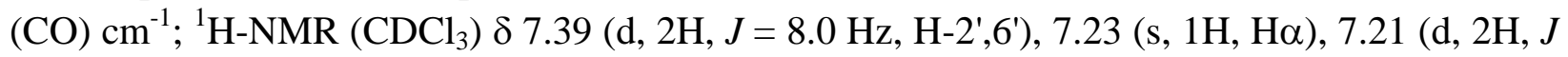
$\left.=8.0 \mathrm{~Hz}, \mathrm{H}-3^{\prime}, 5^{\prime}\right), 5.21$ (q, 1H, J = $\left.7.1 \mathrm{~Hz}, \mathrm{H}-6\right), 2.86$ (s, 3H, $\mathrm{NCH}_{3}$ ), 2.58 (s, 3H, $\mathrm{COCH}_{3}$ ), 1.49 (d, 3H, $\left.J=7.1 \mathrm{~Hz}, \mathrm{C}_{6}-\mathrm{CH}_{3}\right) ;{ }^{13} \mathrm{C}-\mathrm{NMR}\left(\mathrm{CDCl}_{3}\right) \delta 171.6\left(\mathrm{COCH}_{3}\right), 168.2(\mathrm{C}-5), 163.3(\mathrm{C}-2)$, 134.5 (C-4'), 130.8 (C-2',6'), 123.1 (C-3), 131.9 (C-1'), 131.3 (C $\alpha), 129.1$ (C-3',5'), 52.8 (C-6), $34.8\left(\mathrm{NCH}_{3}\right), 27.2\left(\mathrm{COCH}_{3}\right), 18.4\left(\mathrm{C}_{6}-\underline{\mathrm{CH}}_{3}\right)$. Anal. Calc. for $\mathrm{C}_{15} \mathrm{H}_{15} \mathrm{ClN}_{2} \mathrm{O}_{3}: \mathrm{C}, 58.73 ; \mathrm{H}, 4.93$; N, 9.13. Found: C, 58.93; H, 5.06; N, 9.09.

Compound (E)-14. Yield 0.006 g, 4\%; yellow solid (after chromatography eluting with 1:8 $\mathrm{Cl}_{2} \mathrm{CH}_{2}$ /petroleum ether); $[\alpha]_{\mathrm{D}}{ }^{25}=-52.8$ (c 0.25, $\mathrm{CHCl}_{3}$ ); IR (KBr) $\vee 1693.3(\mathrm{CO}) \mathrm{cm}^{-1} ;{ }^{1} \mathrm{H}-$

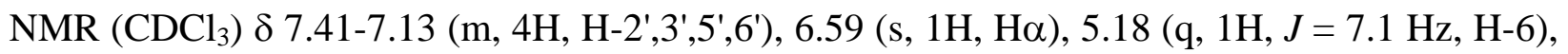
3.28 (s, 3H, $\mathrm{NCH}_{3}$ ), 2.49 (s, 3H, $\mathrm{COCH}_{3}$ ), 1.50 (d, 3H, $J=7.1 \mathrm{~Hz}, \mathrm{C}_{6}-\mathrm{CH}_{3}$ ). Anal. Calc. for $\mathrm{C}_{15} \mathrm{H}_{15} \mathrm{Cl} \mathrm{N} \mathrm{N}_{2}$ : C, 58.73; H, 4.93; N, 9.13. Found: C, 58.89; H, 5.00; N, 9.10.

\section{General procedure for the catalytic hydrogenation of compounds (1)}

A suspension of the suitable compound 1 and 10\% Pd-C in EtOH was hydrogenated at 40 psi for $6 \mathrm{~h}$. After filtering off the Pd-C through a layer of celite, the solvent was evaporated and the residue was purified by fast chromatography on silica gel, eluting with a 1:6 ethyl acetatepetroleum ether mixture.

\section{(4S,1S)-1-(4-Chlorobenzyl)-2,4-dihydro-2,4-dimethyl-1H-pyrazino[2,1-b]quinazoline-3,6-}

dione (3a) and (4S, 1S)-1-(4-chlorobenzyl)-2,4,7,8,9,10-hexahydro-2,4-dimethyl-1Hpyrazino[2,1-b]quinazoline-3,6-dione (15a). Obtained following the general procedure from 1a $(0.100 \mathrm{~g}, 0.273 \mathrm{mmol})$ and $10 \% \mathrm{Pd}-\mathrm{C}(0.016 \mathrm{~g})$ in EtOH $(10 \mathrm{~mL})$.

Compound (3a). Yield 0.063 g, 63\%; yellow solid; mp 96-98 ${ }^{\circ} \mathrm{C}$; IR (KBr) v 1668.0 (CO) cm ${ }^{-1}$; ${ }^{1} \mathrm{H}-\mathrm{NMR}\left(\mathrm{CDCl}_{3}\right) \delta 8.26$ (d, 1H, $\left.J=8.0 \mathrm{~Hz}, \mathrm{H}-7\right), 7.78$ (t, 1H, $\left.J=7.9 \mathrm{~Hz}, \mathrm{H}-9\right), 7.65$ (d, $1 \mathrm{H}, J=$ $8.0 \mathrm{~Hz}, \mathrm{H}-10$ ), 7.49 (t, 1H, $J=7.9 \mathrm{~Hz}, \mathrm{H}-8$ ), 7.25 (d, 2H, $J=7.4 \mathrm{~Hz}, \mathrm{H}-2$ ',6'), 7.09 (d, 2H, $J=7.4$ Hz, H-3',5'), 5.16 (q, 1H, $J=7.1 \mathrm{~Hz}, \mathrm{H}-4), 4.79$ (dd, $1 \mathrm{H}, J=1.4$ and $J=13.1 \mathrm{~Hz}, \mathrm{H}-1$ ), 3.42-3.33

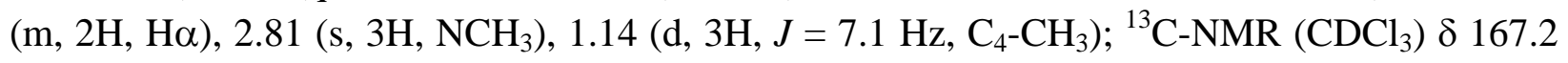
(C-3), 160.4 (C-6), 150.3 (C-11a), 147.3 (C-10a), 135.4 (C-1'), 134.9 (C-9), 129.9 (C-2',6'), 129.3 (C-3',5'), 127.9 (C-4'), 127.2 (C-7), 127.0 (C-8), 126.9 (C-10), 120.3 (C-6a), 65.2 (C-1), 52.4 (C-4), $41.6(\mathrm{C} \alpha), 34.1\left(\mathrm{NCH}_{3}\right), 18.4\left(\mathrm{C}_{4}-\underline{\mathrm{CH}_{3}}\right)$. Anal. Calc. for $\mathrm{C}_{20} \mathrm{H}_{18} \mathrm{ClN}_{3} \mathrm{O}_{2}: \mathrm{C}, 65.31 ; \mathrm{H}$, 4.93; N, 11.42. Found: C, 65.48; H, 4.96; N, 10.43.

Compound (15a). Yield 0.017 g, 17\%; yellow solid; mp 94-96 ${ }^{\circ} \mathrm{C}$; IR (KBr) v $1663.7(\mathrm{CO}) \mathrm{cm}^{-}$ 1; ${ }^{1} \mathrm{H}-\mathrm{NMR}\left(\mathrm{CDCl}_{3}\right) \delta 7.25$ (d, 2H, $J=7.4 \mathrm{~Hz}, \mathrm{H}-2$ ',6'), 7.04 (d, 2H, $J=7.4 \mathrm{~Hz}, \mathrm{H}-3$ ',5'), 4.99 (q, 


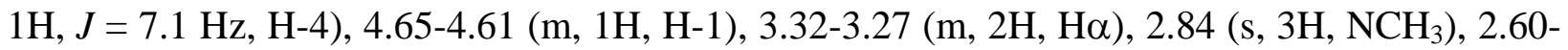
2.49 (m, 4H, H-7,10), 1.80-1.68 (m, 4H, H-8,9), 1.07 (d, 3H, $J=7.1 \mathrm{~Hz}, \mathrm{C}_{4}-\mathrm{CH}_{3}$ ); ${ }^{13} \mathrm{C}-\mathrm{NMR}$ $\left(\mathrm{CDCl}_{3}\right) \delta 167.2$ (C-3), 160.5 (C-6), 159.5 (C-11a), 151.2 (C-10a), 135.3 (C-1'), 129.8 (C-2',6'), 129.3 (C-3',5'), 127.9 (C-4'), 120.1 (C-6a), 64.4 (C-1), 51.9 (C-4), 41.2 (Ca), $33.7\left(\mathrm{NCH}_{3}\right), 31.2$ (C-9), 22.5 (C-7), 21.9 (C-8), 21.5 (C-10), $18.1\left(\mathrm{C}_{4}-\mathrm{CH}_{3}\right)$. Anal. Calc. for $\mathrm{C}_{20} \mathrm{H}_{22} \mathrm{ClN}_{3} \mathrm{O}_{2}$ : C, 64.60; H, 5.96; N, 11.30. Found: C, 64.53; H, 5.98; N, 11.33.

(4S,1S)-1-(4-Chlorobenzyl)-2,4-dihydro-4-methyl-2-phenetyl-1H-pyrazino[2,1-b]quinazoline-3,6dione (3c) and (4S,1S)-1-(4-chlorobenzyl)-2,4,7,8,9,10-hexahydro-4-methyl-2-phenetyl-1Hpyrazino[2,1-b]quinazoline-3,6-dione (15c). Obtained following the general procedure from 1c (0.027 g, $0.059 \mathrm{mmol})$ and 10\% Pd-C (0.003 g) in EtOH (1 mL).

Compound (3c). Yield, 0.014 (51\%), as a colorless oil; IR (KBr) $v 1669.3$ (CO) $\mathrm{cm}^{-1}$; ${ }^{1} \mathrm{H}-\mathrm{NMR}$ $\left(\mathrm{CDCl}_{3}\right) \delta 8.30(\mathrm{~d}, 1 \mathrm{H}, J=8.0 \mathrm{~Hz}, \mathrm{H}-7), 7.80(\mathrm{t}, 1 \mathrm{H}, J=7.9 \mathrm{~Hz}, \mathrm{H}-9), 7.64$ (d, 1H, $J=7.9 \mathrm{~Hz}$, $\mathrm{H}-10$ ), 7.52 (t, 1H, $J=8.0 \mathrm{~Hz}, \mathrm{H}-8), 7.15-7.04$ (m, 4H, H-2',3',5',6'), 7.30 (m, 5H, $\mathrm{CH}_{2} \mathrm{CH}_{2} \underline{\mathrm{Ph}}$ ), 5.21 (q, $1 \mathrm{H}, J=7.1 \mathrm{~Hz}, \mathrm{H}-4)$, 4.60-4.51 (m, 1H, H-1), 4.25-4.14 (m, 1H, $\mathrm{C}_{2} \mathrm{CH}_{2} \mathrm{Ph}$ ), 3.38-3.22 (m, $2 \mathrm{H}, \mathrm{H \alpha}$ ), 2.84-2.78 (m, 1H, $\mathrm{CH}_{2} \mathrm{CH}_{2} \mathrm{Ph}$ ), 2.65-2.62 (m, 2H, $\mathrm{CH}_{2} \mathrm{CH}_{2} \mathrm{Ph}$ ), 1.32 (d, 3H, J = 7.1 $\left.\mathrm{Hz}, \mathrm{C}_{4}-\mathrm{CH}_{3}\right) ;{ }^{13} \mathrm{C}-\mathrm{NMR}\left(\mathrm{CDCl}_{3}\right) \delta 165.8$ (C-3), 159.0 (C-6), 149.6 (C-11a), 146.0 (C-10a), 134.2 (C-9), 136.7 (C-1'), 133.6 (C-4'), 129.4 and 129.1 (C-2',3',5',6'), 128.5 and 127.9 (C2”,3”,5”,6”), 127.4 (C-1”), 127.4 (C-7), 126.6 (C-8), 126.3 (C-4”), 125.9 (C-10), 119.0 (C-6a), 62.2 (C-1), 51.2 (C-4), $40.9(\mathrm{C} \alpha), 46.7\left(\underline{\mathrm{CH}}_{2} \mathrm{CH}_{2} \mathrm{Ph}\right), 32.0\left(\mathrm{CH}_{2} \underline{\mathrm{CH}_{2}} \mathrm{Ph}\right), 17.0\left(\mathrm{C}_{4}-\underline{\mathrm{CH}_{3}}\right) \mathrm{ppm}$. Anal. Calc. for $\mathrm{C}_{27} \mathrm{H}_{24} \mathrm{ClN}_{3} \mathrm{O}_{2}$ : C, 70.81; H, 5.28; N, 9.18. Found: C, 70.65; H, 5.01; N, 8.98.

Compound (15c). Yield, $0.021 \mathrm{~g}$ (44\%); colorless oil; IR (KBr) v 1663.6 (CO) $\mathrm{cm}^{-1}$; ${ }^{1} \mathrm{H}-\mathrm{NMR}$ $\left(\mathrm{CDCl}_{3}\right) \delta$ 7.30-7.02 (m, 9H, H-2',3',5',6',Ph), 5.07 (q, 1H, J = 7.1 Hz, H-4), 4.44-4.39 (m, 1H, H1), 4.20-4.09 (m, 1H, $\underline{\mathrm{C}}_{2} \mathrm{CH}_{2} \mathrm{Ph}$ ), 3.25-3.11 (m, $2 \mathrm{H}, \mathrm{H \alpha}$ ), 2.81-2.74 (m, 1H, $\mathrm{C}_{2} \mathrm{CH}_{2} \mathrm{Ph}$ ), 2.602.49 (m, 6H, $\left.\mathrm{CH}_{2} \underline{\mathrm{C}}_{2} \mathrm{Ph}, \mathrm{H}-7,10\right)$, 1.84-1.74 (m, 4H, H-8,9), 1.24 (d, 3H, J = 7.1 Hz, C $4-\underline{\mathrm{C}}_{3}$ ); ${ }^{13} \mathrm{C}-\mathrm{NMR}\left(\mathrm{CDCl}_{3}\right) \delta 166.9$ (C-3), 160.4 (C-6), 159.7 (C-11a); 141.1 (C-10a), 137.9 (C-1”), 135.2 (C-1'), 129.6 and 129.0 (C-2',3',5’,6'); 128.6 and 128.5 (C-2”,3”,5”,6”), 127.7 (C-4'); 126.5 (C4”); 119.9 (C-6a), 62.9 (C-1), 52.1 (C-4), 41.9 (C $\alpha), 47.7$ ( $\left.\mathrm{CH}_{2} \mathrm{CH}_{2} \mathrm{Ph}\right), 33.1\left(\mathrm{CH}_{2} \underline{\mathrm{CH}}{ }_{2} \mathrm{Ph}\right), 31.7$ (C-9), 22.2 (C-7), 22.0 (C-8), 21.6 (C-10), $18.0\left(\mathrm{C}_{4}-\underline{\mathrm{CH}_{3}}\right)$ ppm. Anal. Calcd. for $\mathrm{C}_{27} \mathrm{H}_{28} \mathrm{ClN}_{3} \mathrm{O}_{2}$ : C, 70.19; H, 6.11; N, 9.10. Found: C, 69.90; H, 5.92; N, 8.98.

(4S,1S)-1-(4-Bromobenzyl)-2,4-dihydro-2,4-dimethyl-1H-pyrazino[2,1-b]quinazoline-3,6dione (3d). Obtained following the general procedure from 1d $(0.100 \mathrm{~g}, 0.243 \mathrm{mmol})$ and 10\% Pd-C (0.014 g) in EtOH (10 mL). Yield 0.055 g, 56\%; reddish solid; mp 140-142 ${ }^{\circ} \mathrm{C}$; IR (KBr) v 1720.2 and 1666.1 (CO) $\mathrm{cm}^{-1}$; ${ }^{1} \mathrm{H}-\mathrm{NMR}$ (DMSO- $d_{6}$ ) $\delta 8.11$ (d, $1 \mathrm{H}, J=8.0 \mathrm{~Hz}, \mathrm{H}-7$ ), 7.96 (t, $1 \mathrm{H}$, $J=7.9 \mathrm{~Hz}, \mathrm{H}-9), 7.68$ (d, 1H, $J=8.0 \mathrm{~Hz}, \mathrm{H}-10), 7.54$ (t, 1H, $J=7.9 \mathrm{~Hz}, \mathrm{H}-8), 7.23$ (d, 2H, $J=$ $\left.7.3 \mathrm{~Hz}, \mathrm{H}-2^{\prime}, 6^{\prime}\right), 7.02$ (d, 2H, $\left.J=7.3 \mathrm{~Hz}, \mathrm{H}-3 ', 5 '\right), 5.03-4.99$ (m, 1H, H-1), 4.75 (q, 1H, $J=7.1$

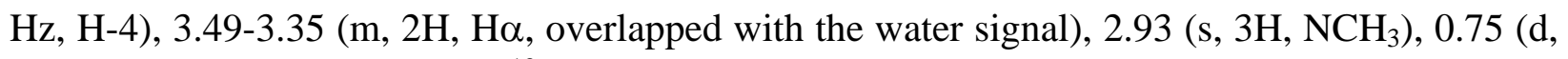
$\left.3 \mathrm{H}, J=7.1 \mathrm{~Hz}, \mathrm{C}_{4}-\mathrm{CH}_{3}\right) \mathrm{ppm} ;{ }^{13} \mathrm{C}-\mathrm{NMR}$ (DMSO-d $)_{6} \delta 167.4$ (C-3), 161.2 (C-6), 152.3 (C-11a), 148.5 (C-10a), 137.5 (C-1'), 136.4 (C-9), 131.4 (C-2',6'), 130.2 (C-3',5'), 128.8 (C-4'), 128.4 (C7), 128.5 (C-8), 127.7 (C-10), 121.2 (C-6a), 64.9 (C-1), 53.1 (C-4), 39.7 (C $\alpha), 34.2\left(\mathrm{NCH}_{3}\right), 19.1$ 
$\left(\mathrm{C}_{4}-\mathrm{CH}_{3}\right)$. Anal. Calc. for $\mathrm{C}_{20} \mathrm{H}_{18} \mathrm{BrN}_{3} \mathrm{O}_{2}$ : C, 58.26; H, 4.40; N, 10.19. Found: C, 58.36; H, 4.42; $\mathrm{N}, 10.17$.

(4S,1S)-1-(3,4-Dichlorobenzyl)-2,4-dihydro-2,4-dimethyl-1H-pyrazino[2,1-b]quinazoline-

3,6-dione (3f). Obtained following the general procedure from $1 \mathbf{f}(0.035 \mathrm{~g}, 0.087 \mathrm{mmol})$ and $10 \%$ Pd-C (0.005 g) in EtOH (2 mL). Yield 0.014 g, 41\%; yellow solid; mp 190-192 ${ }^{\circ} \mathrm{C}$; IR $(\mathrm{KBr}) \vee 1661.1(\mathrm{CO}) \mathrm{cm}^{-1} ;{ }^{1} \mathrm{H}-\mathrm{NMR}\left(\mathrm{CDCl}_{3}\right) \delta 8.29(\mathrm{~d}, 1 \mathrm{H}, J=8.0 \mathrm{~Hz}, \mathrm{H}-7), 7.81(\mathrm{t}, 1 \mathrm{H}, J=7.9$ Hz, H-9), 7.63 (d, 1H, $J=8.0 \mathrm{~Hz}, \mathrm{H}-10$ ), 7.52 (t, 1H, $J=7.9 \mathrm{~Hz}, \mathrm{H}-8$ ), 7.38 (d, $1 \mathrm{H}, J=8.0 \mathrm{~Hz}$, H-5'), 7.30 (d, 1H, $\left.J=2.0 \mathrm{~Hz}, \mathrm{H}-2^{\prime}\right), 7.00$ (d, 1H, $J=8.0 \mathrm{~Hz}, \mathrm{H}-6^{\prime}$ ), 5.27 (q, $1 \mathrm{H}, J=7.1 \mathrm{~Hz}, \mathrm{H}-$ 4), 4.76 (dd, $1 \mathrm{H}, J=1.9 \mathrm{~Hz}$ and $J=7.2 \mathrm{~Hz}, \mathrm{H}-1$ ), 3.37-3.25 (m, 2H, $\mathrm{H \alpha}$ ), 2.92 (s, 3H, $\mathrm{NCH}_{3}$ ), 1.46 (d, 3H, $\left.J=7.1 \mathrm{~Hz}, \mathrm{C}_{4}-\mathrm{CH}_{3}\right) ;{ }^{13} \mathrm{C}-\mathrm{NMR}\left(\mathrm{CDCl}_{3}\right) \delta 167.5$ (C-3), 160.6 (C-6), 149.9 (C-11a), 147.3 (C-10a), 136.3 (C-1'), 135.3 (C-9), 133.6 (C-2'), 132.5 (C-3'), 131.8 (C-5'), 131.4 (C-6'), 129.4 (C-4'), 127.7 (C-7), 127.3 (C-8), 127.2 (C-10), 120.6 (C-6a), 65.3 (C-1), 52.6 (C-4), 41.7 $(\mathrm{C} \alpha)$, $34.7\left(\mathrm{NCH}_{3}\right), 19.3\left(\mathrm{C}_{4}-\mathrm{CH}_{3}\right)$. Anal. Calc. for $\mathrm{C}_{20} \mathrm{H}_{17} \mathrm{Cl}_{2} \mathrm{~N}_{3} \mathrm{O}_{2}: \mathrm{C}, 59.71 ; \mathrm{H}, 4.26 ; \mathrm{N}, 10.45$. Found: C, 59.84; H, 4.25; N, 10.48.

(4S,1S)-1-(2,4-Difluorobenzyl)-2,4-dihydro-2,4-dimethyl-1H-pyrazino[2,1-b]quinazoline-

3,6-dione (3g). Obtained following the general procedure from $\mathbf{1 g}(0.035 \mathrm{~g}, 0.095 \mathrm{mmol})$ and $10 \%$ Pd-C $(0.005 \mathrm{~g})$ in EtOH (2 mL). Yield, $0.011 \mathrm{~g}, 30 \%$ (50\% yield based on unrecovered starting material); colourless oil; IR $(\mathrm{KBr}) \vee 1673.9(\mathrm{CO}) \mathrm{cm}^{-1} ;{ }^{1} \mathrm{H}-\mathrm{NMR}\left(\mathrm{CDCl}_{3}\right) \delta 8.28(\mathrm{~d}, 1 \mathrm{H}$, $J=8.0 \mathrm{~Hz}, \mathrm{H}-7$ ), 7.77 (t, 1H, $J=8.0 \mathrm{~Hz}, \mathrm{H}-9$ ), 7.57-7.58 (m, 2H, H-8,10), 7.08 (q, 1H, $J=7.6$ Hz, H-5'), 6.75-6.85 (m, 2H, H-3',6'); 5.28 (q, 1H, J = 7.1 Hz, H-4), 4.76-4.71 (m, 1H, H-1), 3.46-3.29 (m, 2H, Ha), 2.98 (s, 3H, NCH $), 1.54$ (d, 3H, $\left.J=7.1 \mathrm{~Hz}, \mathrm{C}_{4}-\mathrm{CH}_{3}\right) ;{ }^{13} \mathrm{C}-\mathrm{NMR}$ (CDCl3) $\delta 165.2$ (C-3), 160.8 (dd, $J=250.2$ and $12.1 \mathrm{~Hz}, \mathrm{C}-2$ '), 158.4 (C-6), 159.7 (dd, $J=$ 249.0 and $11.8 \mathrm{~Hz}, \mathrm{C}-4$ ), 147.5 (C-11a), 145.0 (C-10a), 132.9 (C-9), 132.7 (dd, $J=9.7$ and 9.6 Hz, C-6'), 125.4 (C-7), 124.9 (C-8), 124.9 (C-10), 118.3 (C-6a), 116.6 (dd, $J=15.9$ and 3.9 Hz, C-1'), 110.0 (dd, $J=21.2$ and 3.7 Hz, C-5'), 102.3 (t, $J=25.8$ Hz, C-3'), 64.3 (C-1), 52.7 (C-4), $35.1(\mathrm{C} \alpha)$, $34.3\left(\mathrm{NCH}_{3}\right), 19.1\left(\mathrm{C}_{4}-\mathrm{CH}_{3}\right)$. Anal. Calc. for $\mathrm{C}_{20} \mathrm{H}_{17} \mathrm{~F}_{2} \mathrm{~N}_{3} \mathrm{O}_{2}$ : C, 65.03; H, 4.64; N, 11.38. Found: C, 64.95; H, 4.66; N, 11.40 .

(4S,1S)-1-[(1-Benzyl-3-indolyl)methyl]-2,4-dihydro-2,4-dimethyl-1H-pyrazino[2,1-b]quinazoline-

3,6-dione (3h). Obtained following the general procedure from $\mathbf{1 h}(0.100 \mathrm{~g}, 0.217 \mathrm{mmol})$ and $10 \%$ Pd-C (0.012 g) in EtOH (10 mL). Yield, $0.056 \mathrm{~g}$, 86\%; pale yellow oil; IR (KBr) $\vee 1665.3$ (CO) $\mathrm{cm}^{-1} ;{ }^{1} \mathrm{H}-\mathrm{NMR}\left(\mathrm{CDCl}_{3}\right) \delta 8.25$ (d, $\left.1 \mathrm{H}, J=7.9 \mathrm{~Hz}, \mathrm{H}-7\right), 7.81$ (t, $\left.1 \mathrm{H}, J=7.9 \mathrm{~Hz}, \mathrm{H}-9\right), 7.41$ (d, 1H, $J=7.9 \mathrm{~Hz}, \mathrm{H}-10$ ), 7.50 (t, 1H, $J=7.9 \mathrm{~Hz}, \mathrm{H}-8$ ), 7.33-7.20 (m, 5H, Ph), 7.13-7.06 (m, 2H, H-2',4'), 6.99-6.94 (m, 2H, H-5',6'), 7.72 (d, 1H, $J=7.9, \mathrm{~Hz}, \mathrm{H}-7$ '), 5.24 (s, 2H, $\mathrm{CH}_{2} \mathrm{Ph}$ ), 5.14 (q,

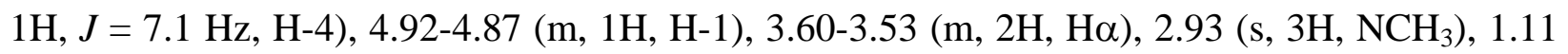
(d, 3H, $\left.J=7.1 \mathrm{~Hz}, \mathrm{C}_{4}-\mathrm{CH}_{3}\right) ;{ }^{13} \mathrm{C}-\mathrm{NMR}\left(\mathrm{CDCl}_{3}\right) \delta 167.5$ (C-3), 160.6 (C-6), 151.2 (C-11a), 144.8 (C-10a), 137.4 (C-7a'), 133.8 (C-9), 129.2, 127.4, 127.3, 127.2 (Ph), 128.4 (C-2'), 128.3 (C-7), 128.2 (C-8), 127.9 (C-10), 127.1 (C-4'), 122.7 (C-5'), 120.3 (C-6'), 119.0 (C-6a), 110.4 (C-7'),

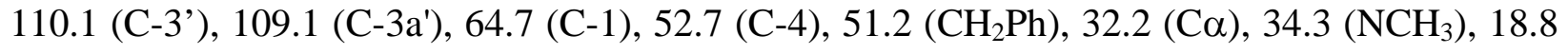
$\left(\mathrm{C}_{4}-\mathrm{CH}_{3}\right)$. Anal. Calc. for $\mathrm{C}_{29} \mathrm{H}_{26} \mathrm{~N}_{4} \mathrm{O}_{2}$ : C, 75.30; H, 5.67; N, 12.11. Found: C, 75.41; H, 5.64; N, 12.14. 
(4S,1S)-2,4-Dihydro-2,4-dimethyl-1-[(1-tosyl-3-indolyl)methyl]-1H-pyrazino[2,1-b]quinazoline-3,6dione (3i). Obtained following the general procedure from $1 \mathbf{i}(0.080 \mathrm{~g}, 0.152 \mathrm{mmol})$ and 10\% PdC (0.009 g) in EtOH (6 mL). Yield, 0.061 g, 77\%; pale yellow oil; IR (KBr) v 1669.9 (CO) cm ${ }^{-1}$; ${ }^{1} \mathrm{H}-\mathrm{NMR}\left(\mathrm{CDCl}_{3}\right) \delta 8.26$ (d, $\left.1 \mathrm{H}, J=8.0 \mathrm{~Hz}, \mathrm{H}-7\right), 7.99$ (d, $1 \mathrm{H}, J=8.0 \mathrm{~Hz}, \mathrm{H}-7$ '), 7.84-7.78 (m, 2H, H-9,10), 7.52 (t, 1H, J = 7.9 Hz, H-8), 7.74-7.76 (m, 3H, H-4',2",6"), 7.44 (s, 1H, H-2'), 7.32 (t, 1H, $J=7.6 \mathrm{~Hz}, \mathrm{H}-6$ '), 7.20 (d, 2H, $J=7.5 \mathrm{~Hz}, \mathrm{H}-3 ", 5 "), 7.12$ (t, 1H, $J=7.6 \mathrm{~Hz}, \mathrm{H}-5$ '), 5.11 (q,

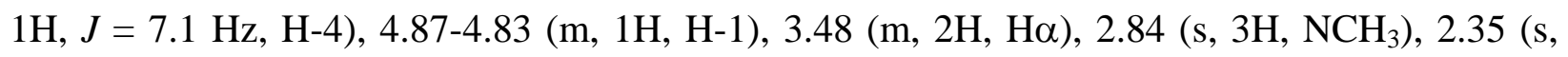
$\left.3 \mathrm{H}, \mathrm{CH}_{3}-\mathrm{C}_{6} \mathrm{H}_{4}\right), 0.99$ (d, 3H, $\left.J=7.1 \mathrm{~Hz}, \mathrm{C}_{4}-\mathrm{CH}_{3}\right) ;{ }^{13} \mathrm{C}-\mathrm{NMR}\left(\mathrm{CDCl}_{3}\right) \delta 165.7$ (C-3), 159.1 (C-6), 149.6 (C-11a), 148.9 (C-4"), 145.9 (C-7'a), 144.1 (C-10a), 133.9 (C-9), 129.0 (C-2'), 128.9 (C1"), 128.8 (C-2",6"), 126.2 (C-7), 125.8 (C-8), 125.6 (C-3",5"), 125.5 (C-10), 126.2 (C-4'), 123.9 (C-5'), 122.4 (C-6'), 119.6 (C-6a), 115.2 (C-7'), 112.7 (C-3'a), 110.0 (C-3’), 63.9 (C-1), 52.4 (C4), $32.0(\mathrm{C} \alpha), 34.3\left(\mathrm{NCH}_{3}\right), 22.0\left(\underline{\mathrm{CH}}_{3}-\mathrm{C}_{6} \mathrm{H}_{4}\right), 18.8\left(\mathrm{C}_{4}-\underline{\mathrm{CH}}_{3}\right)$. Anal. Calc. for $\mathrm{C}_{29} \mathrm{H}_{26} \mathrm{~N}_{4} \mathrm{O}_{4} \mathrm{~S}$ : C, 66.14; H, 4.98; N, 10.64. Found: C, 65.95; H, 5.00; N, 10.66.

(4S,1S)-2,4-Dihydro-1-(4-methoxybenzyl)-2,4-dimethyl-1H-pyrazino[2,1-b]quinazoline-3,6dione (3j) and (4S,1S)-2,4,7,8,9,10-hexahydro-1-(4-methoxybenzyl)-2,4-dimethyl-1Hpyrazino[2,1-b]quinazoline-3,6-dione (15j). Obtained following the general procedure from $\mathbf{1 j}$ (0.107 g, $0.296 \mathrm{mmol})$ and 10\% Pd-C (0.017 g) in EtOH (10 mL), which afforded $\mathbf{3 j}+\mathbf{1 5 j}$ after $6 \mathrm{~h}$ reaction time. Starting from $\mathbf{1 j}(0.101 \mathrm{~g}, 0.279 \mathrm{mmol})$ and $10 \% \mathrm{Pd}-\mathrm{C}(0.016 \mathrm{~g})$ in EtOH (10 $\mathrm{mL}$ ), 15j was the only product after $16 \mathrm{~h}$. Yield, $0.051 \mathrm{~g}, 48 \%$ (together with $30 \%$ of $\mathbf{1 5 j}$ after 6 h); yellow oil; IR (KBr) v $1663.8(\mathrm{CO}) \mathrm{cm}^{-1}$; ${ }^{1} \mathrm{H}-\mathrm{NMR}\left(\mathrm{CDCl}_{3}\right) \delta 8.25$ (d, $\left.1 \mathrm{H}, J=8.0 \mathrm{~Hz}, \mathrm{H}-7\right)$, 7.76 (t, 1H, $J=7.9 \mathrm{~Hz}, \mathrm{H}-9$ ), 7.64 (d, 1H, $J=8.0 \mathrm{~Hz}, \mathrm{H}-10$ ), 7.46 (t, 1H, $J=7.9 \mathrm{~Hz}, \mathrm{H}-8$ ), 6.98 (d, 2H, $J=7.5$ Hz, H-2',6'), 6.77 (d, 2H, $J=7.5$ Hz, H-3',5'), 5.15 (q, 1H, J = 7.1 Hz, H-4), 4.75-

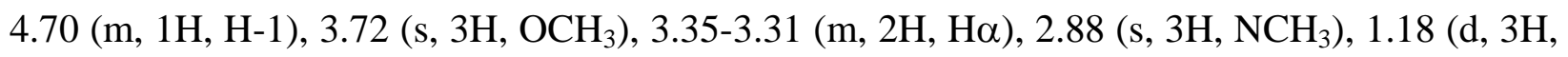
$\left.J=7.1 \mathrm{~Hz}, \mathrm{C}_{4}-\underline{\mathrm{C}}_{3}\right) \mathrm{ppm} ;{ }^{13} \mathrm{C}-\mathrm{NMR}\left(\mathrm{CDCl}_{3}\right) \delta 167.2$ (C-3), 160.4 (C-6), 150.4 (C-4'), 147.3 (C10a,11a), 134.7 (C-9), 130.6 (C-2',6'), 127.1 (C-1'), 127.0 (C-7), 126.8 (C-8), 126.7 (C-10), 120.1 (C-6a), 114.4 (C-3',5'), 65.2 (C-1), $55.4\left(\mathrm{OCH}_{3}\right), 52.4$ (C-4), $40.8(\mathrm{C \alpha}), 34.2\left(\mathrm{NCH}_{3}\right), 18.4$ $\left(\mathrm{C}_{4}-\underline{C H}_{3}\right)$. Anal. Calc. for $\mathrm{C}_{21} \mathrm{H}_{21} \mathrm{~N}_{3} \mathrm{O}_{3}$ : C, 69.41; H, 5.82; N, 11.56. Found: C, 69.36; H, 5.79; N, 11.59 .

Data for (15j). Yield, 0.085 g, 83\%; yellow solid; mp 138-140 ${ }^{\circ} \mathrm{C}$; IR (KBr) v 1663.1 (CO) $\mathrm{cm}^{-1}$; ${ }^{1} \mathrm{H}-\mathrm{NMR}\left(\mathrm{CDCl}_{3}\right) \delta 6.92$ (d, 2H, $J=7.8 \mathrm{~Hz}, \mathrm{H}-2$ ',6'), 6.77 (d, 2H, $\left.J=7.8 \mathrm{~Hz}, \mathrm{H}-3^{\prime}, 5^{\prime}\right), 4.96$ (q,

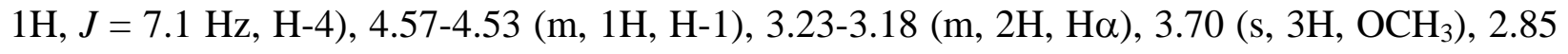
(s, 3H, NCH 3 ), 2.57-2.45 (m, 4H, H-7,10), 1.78-1.75 (m, 4H, H-8,9), 1.05 (d, 3H, J = 7.1 Hz, C4$\left.\mathrm{CH}_{3}\right) ;{ }^{13} \mathrm{C}-\mathrm{NMR}\left(\mathrm{CDCl}_{3}\right) \delta 167.1$ (C-3), 160.5 (C-6), 151.3 (C-4'), 143.9 (C-11a), 143.8 (C-10a), 130.8 (C-2',6'), 127.3 (C-1'), 120.0 (C-6a), 114.6 (C-3',5'), 64.8 (C-1), $55.4\left(\mathrm{OCH}_{3}\right), 52.1$ (C-4),

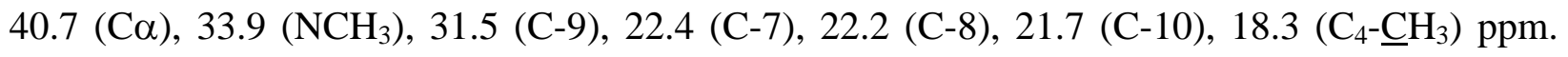
Anal. Calc. for $\mathrm{C}_{21} \mathrm{H}_{25} \mathrm{~N}_{3} \mathrm{O}_{3}$ : C, 68.64; H, 6.86; N, 11.44. Found: C, 68.53; H, 6.81; N, 11.46 .

(4S,1S)-1-(4-Chlorobenzyl)-2,4-dihydro-4-methyl-1H-pyrazino[2,1-b]quinazoline-3,6-dione (3k), (4S,1S)-1(4-chlorobenzyl)-2,4,7,8,9,10-hexahydro-4-methyl-1H-pyrazino[2,1-b]quinazoline-3,6-dione (15k) and (4S,1R)-1-(4-chlorobenzyl)-2,4-dihydro-4-methyl-1H-pyrazino[2,1-b]quinazoline-3,6-dione (16). Obtained 
following the general procedure from $1 \mathbf{k}(0.030 \mathrm{~g}, 0.085 \mathrm{mmol})$ and $10 \% \mathrm{Pd}-\mathrm{C}(0.005 \mathrm{~g})$ in $\mathrm{EtOH}(2 \mathrm{~mL})$.

Compound (3k). Yield, 0.009 g, 30\%; colorless oil; IR (KBr) v 2930.5 (NH), 1686.8 (CO) cm-1; ${ }^{1} \mathrm{H}-\mathrm{NMR}\left(\mathrm{CDCl}_{3}\right) \delta 8.31(\mathrm{~d}, 1 \mathrm{H}, J=8.0 \mathrm{~Hz}, \mathrm{H}-7), 7.81$ (t, $\left.1 \mathrm{H}, J=7.9 \mathrm{~Hz}, \mathrm{H}-9\right), 7.72(\mathrm{~d}, 1 \mathrm{H}, J=$ 8.0 Hz, H-10), 7.53 (t, 1H, J = 7.9 Hz, H-8), 7.38-7.23 (m, 4H, H-2',3',5',6'), 6.46 (br s, 1H, NH), 5.24 (q, 1H, $J=7.1 \mathrm{~Hz}, \mathrm{H}-4), 4.86-4.79$ (m, 1H, H-1), 3.48 (dd, $1 \mathrm{H}, J=13.4$ and $J=3.7 \mathrm{~Hz}$, $\mathrm{H \alpha}$ ), 3.17 (dd, $1 \mathrm{H}, J=13.4$ and $J=10.6 \mathrm{~Hz}, \mathrm{H \alpha}$ ), 1.49 (d, $3 \mathrm{H}, J=7.1 \mathrm{~Hz}, \mathrm{C}_{4}-\mathrm{CH}_{3}$ ); ${ }^{13} \mathrm{C}-\mathrm{NMR}$ $\left(\mathrm{CDCl}_{3}\right) \delta 167.0$ (C-3), 159.1 (C-6), 149.0 (C-11a), 148.0 (C-10a), 134.0 (C-4'), 133.3 (C-9), 128.1 (C-2',6'), 127.9 (C-1'), 127.8 (C-3',5'), 126.4 (C-7), 126.0 (C-8), 125.5 (C-10), 119.1 (C6a), 55.4 (C-1), 52.5 (C-4), $36.6(\mathrm{C} \alpha), 15.5\left(\mathrm{C}_{4}-\mathrm{CH}_{3}\right)$. Anal. Calcd. for $\mathrm{C}_{19} \mathrm{H}_{16} \mathrm{ClN}_{3} \mathrm{O}_{2}$ : C, 64.50; H, 4.56; N, 11.88. Found: C, 64.39; H, 4.61; N, 11.65.

Compound (15k). Yield, 0.013 g, 33\%; colorless oil; IR (KBr) v 2924.3 (NH), 1681.7 (CO) cm1; ${ }^{1} \mathrm{H}-\mathrm{NMR}\left(\mathrm{CDCl}_{3}\right) \delta$ 7.39-7.19 (m, 4H, H-2',3',5',6'), 6.37 (sa, 1H, NH), 5.09 (q, 1H, $J=7.1$ $\mathrm{Hz}, \mathrm{H}-4)$, 4.69-4.60 (m, 1H, H-1), 3.38 (dd, $1 \mathrm{H}, J=13.5$ and $J=3.8 \mathrm{~Hz}, \mathrm{H \alpha}$ ), 3.05 (dd, $1 \mathrm{H}, J=$ 13.4 and $J=9.8 \mathrm{~Hz}, \mathrm{H \alpha}$ ), 2.67-2.53 (m, 4H, H-7,10), 1.86-1.72 (m, 4H, H-8,9), 1.40 (d, 3H, $J=$ $\left.7.1 \mathrm{~Hz}, \mathrm{C}_{4}-\mathrm{CH}_{3}\right) ;{ }^{13} \mathrm{C}-\mathrm{NMR}\left(\mathrm{CDCl}_{3}\right) \delta 169.9$ (C-3), 161.7 (C-6), 152.8 (C-11a), 152.0 (C-10a), 136.4 (C-1'), 130.9 (C-2',6'), 130.5 (C-3',5'), 129.0 (C-4'), 121.4 (C-6a), 58.0 (C-1), 52.0 (C-4), 44.5 (C $\alpha$ ), 31.8 (C-9), 22.7 (C-7), 22.4 (C-8), 22.0 (C-10), $20.2\left(\mathrm{C}_{4}-\mathrm{CH}_{3}\right)$. Anal. Calc. for $\mathrm{C}_{19} \mathrm{H}_{20} \mathrm{ClN}_{3} \mathrm{O}_{2}$ : C, 63.77; H, 5.63; N, 11.74. Found: C, 63.84; H, 5.69; N, 11.70.

Compound (16). Yield, 0.009 g, 30\%; colorless oil; IR (KBr) v 2925.3 (NH), 1683.5 (CO) cm-1; ${ }^{1} \mathrm{H}-\mathrm{NMR}\left(\mathrm{CDCl}_{3}\right) \delta 8.31$ (d, 1H, $\left.J=8.0 \mathrm{~Hz}, \mathrm{H}-7\right), 7.82-7.75$ (m, 2H, H-9,10), 7.54 (t, 1H, $J=7.9$ Hz, H-8), 7.45-7.29 (m, 4H, H-2',3',5',6'), 5.81 (br s, 1H, NH), 5.47 (q, 1H, J = $6.8 \mathrm{~Hz}, \mathrm{H}-4$ ),

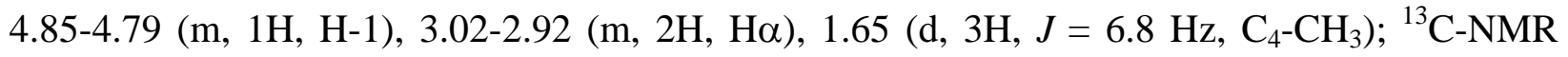
$\left(\mathrm{CDCl}_{3}\right) \delta 165.0$ (C-3), 158.6 (C-6), 148.1 (C-11a), 147.0 (C-10a), 139.7 (C-1'), 133.2 (C-9), 128.0 (C-2',6'), 127.8 (C-3',5'), 127.3 (C-4'), 126.1 (C-7), 125.5 (C-8), 125.3 (C-10), 118.6 (C6a), 58.4 (C-1), 52.3 (C-4), $44.6(\mathrm{C} \alpha), 17.2\left(\mathrm{C}_{4}-\underline{\mathrm{CH}_{3}}\right)$. Anal. Calcd. for $\mathrm{C}_{19} \mathrm{H}_{16} \mathrm{ClN}_{3} \mathrm{O}_{2}: \mathrm{C}, 64.50$; H, 4.56; N, 11.88. Found: C, 64.42; H, 4.41; N, 11.78.

(3S,6S)-3-(4-Methoxybenzylidene)-6-methylpiperazine-2,5-dione (18). Obtained following the general procedure from $17^{12 \mathrm{a}}(0.114 \mathrm{~g}, 0.463 \mathrm{mmol})$ and $10 \% \mathrm{Pd}-\mathrm{C}(0.027 \mathrm{~g})$ in EtOH $(10$ $\mathrm{mL}$ ), in $2 \mathrm{~h}$. Yield $0.083 \mathrm{~g}, 73 \%$; white solid (after chromatography eluting with 1:2 EtOAc/petroleum ether); mp: $225-227^{\circ} \mathrm{C} ;[\alpha]_{\mathrm{D}}{ }^{25}=+17.2$ (c 0.14, DMSO); IR (KBr) $v 3191.2$ and $3052.9(\mathrm{NH}), 1677.1(\mathrm{CO}), 1256.5\left(\mathrm{OCH}_{3}\right) \mathrm{cm}^{-1} ;{ }^{1} \mathrm{H}-\mathrm{NMR}\left(\mathrm{DMSO}-d_{6}\right) \delta 8.09$ (br s, $1 \mathrm{H}, \mathrm{H}-$ 4), 8.01 (br s, 1H, H-1), 7.05 (d, 2H, $J=7.8 \mathrm{~Hz}, \mathrm{H}-2$ ',6'), 6.84 (d, 2H, $J=7.8 \mathrm{~Hz}, \mathrm{H}-3$ ',5'), 4.124.10 (m, 1H, H-3), 3.69 (s, 3H, $\mathrm{OCH}_{3}$ ), 3.01 (q, 1H, $\left.J=7.0 \mathrm{~Hz}, \mathrm{H}-6\right), 3.05$ (dd, 1H, $J=13.4$ and $J=3.2 \mathrm{~Hz}, \mathrm{H \alpha}$ ), 2.77 (dd, $1 \mathrm{H}, J=13.4$ and $J=5.0 \mathrm{~Hz}, \mathrm{H \alpha}$ ), 0.51 (d, 3H, $J=7.0 \mathrm{~Hz}, \mathrm{C}_{6}-\mathrm{CH}_{3}$ ); ${ }^{13}$ C-NMR (DMSO- $d_{6}$ ) $\delta 167.6$ (C-5), 165.8 (C-2), 158.1 (C-4'), 131.3 (C-2',6'), 127.8 (C-1'), 113.4 (C-3',5'), $55.4\left(\mathrm{OCH}_{3}\right), 55.0(\mathrm{C}-3), 49.6(\mathrm{C}-6), 37.3(\mathrm{C} \alpha), 19.7\left(\mathrm{C}_{6}-\underline{\mathrm{CH}}_{3}\right)$. Anal. Calc. for $\mathrm{C}_{13} \mathrm{H}_{16} \mathrm{~N}_{2} \mathrm{O}_{3}$ : C, 62.89; H, 6.50; N, 11.28. Found: C, 62.73; H, 6.49; N, 11.22. 


\section{Acknowledgements}

Financial support from MICINN (grant CTQ2009-12320-BQU) and UCM (Grupos de Investigación, grant 920234) is gratefully acknowledged.

\section{References and Notes}

1. For reviews, see: (a) Mhaske, S. B.; Argade, N. P. Tetrahedron 2006, 62, 9787. (b) Eguchi, S. Top. Heterocycl. Chem. 2006, 6, 113.

2. For a review, see: Avendaño, C.; Menéndez, J. C. Curr. Org. Chem. 2003, 7, 149.

3. (a) Penn, J.; Mantle, P. G.; Bilton, J. N.; Sheppard, R. N. J. Chem. Soc., Perkin Trans. 1 1992, 1495. (b) For the synthesis of both enantiomers of glyantripine, see: Cledera, P.; Avendaño, C.; Menéndez, J. C. J. Org. Chem. 2000, 65, 1743.

4. (a) Numata, A.; Takahashi, C.; Matsushita, T.; Miyamoto, T.; Kawai, K.; Usami, Y.; Matsumura, E.; Inoue, M.; Ohishi, H.; Shingu, T. Tetrahedron Lett. 1992, 33, 1621. (b) Takahashi, C.; Matsushita, T.; Doi, M.; Minoura, K.; Shingu, T.; Kumeda, Y.; Numata A. J. Chem. Soc. Perkin Trans. 1 1995, 2345.

5. (a) Wong, S.-M.; Musza, L. L.; Kydd, G. C.; Kullnig, R.; Gillum, A. M.; Cooper, R. J. Antibiotics 1993, 46, 545. (b) Fujimoto, H.; Negishi, E.; Yamaguchi, K.; Nishi, N.; Yamazaki, M. Chem. Pharm. Bull. 1996, 44, 1843.

6. (a) Hochlowski, J. E., Mullally, M. M.; Spanton, S. G., Whittern, D. N.; Hill, P.; McAlpine, J. B., J. Antibiot. 1993, 46, 380. (b) For the synthesis of $N$-acetylardeemin, see: Depew, K. M.; Marsden, S. P.; Zatorska, D.; Zatorski, A.; Bornmann, W. G.; Danishefsky, S. J. J. Am. Chem. Soc. 1999, 121, 11953.

7. Karwowski, J. P.; Jackson, M.; Rasmussen, R. R.; Humphrey, P. E.; Poddig, J. B.; Kohl, W. L.; Scherr, M. H.; Kadam, S.; McAlpine, J. B., J. Antibiot. 1993, 46, 374.

8. For reviews of MDR inhibitors, see: (a) Avendaño, C.; Menéndez, J. C. Curr. Med. Chem. 2002, 9, 159. (b) Avendaño, C.; Menéndez, J. C. Med. Chem. Rev. Online, 2004, 1, 419.

9. Chou, T. C.; Depew, K. M.; Zheng, Y. H.; Safer, M. L.; Chan, D.; Helfrich, B.; Zatorska, D.; Zatorski, A.; Bornmann, W.; Danishefsky, S. J. Proc. Natl. Acad. Sci. USA 1998, 95, 8369.

10. (a) Méndez-Vidal, C.; Quesada, A. R. Cancer Lett. 1998, 132, 45. (b) Avendaño, C.; Caballero, E.; Méndez-Vidal, C.; Quesada, A. R.; Menéndez, J. C. Lett. Drug Des. Discov. 2006, 3, 369.

11. Chou, T.-C.; Bertino, J. R.; Danishefsky, S. J.; Kahan, B. D. U. S. Patent 6,355,639 (March 12, 2002).

12. (a) Cledera, P.; Avendaño, C.; Menéndez, J. C. Tetrahedron 1998, 54, 12349. (b) Cledera, P.; Sánchez, J. D.; Caballero, E.; Yates, T.; Ramírez, E. G.; Avendaño, C.; Ramos, M. T.; Menéndez, J. C. Synthesis 2007, 3390.

13. Rajappa, S.; Advani, B. G. J. Chem. Soc., Perkin Trans. I 1974, 2122. 
14. (a) Martín-Santamaría, S.; Buenadicha, F. L.; Espada, M.; Söllhuber, M. M.; Avendaño, C. J. Org. Chem. 1997, 62, 6424. (b) Martín-Santamaría, S.; Espada, M.; Avendaño, C. Tetrahedron 1997, 53, 16795. (c) Heredia, M. L.; Fernández, M.; Cuesta, E.; Avendaño, C. Tetrahedron: Asymmetry 2001, 12, 411.

15. For one earlier, isolated example of this reaction, see: Heredia, M. L.; Cuesta, E.; Avendaño, C. Tetrahedron, 2001, 57, 1987.

16. For a review of the captodative effect in $\alpha$-amino acids, see: Easton, C. J. Chem. Rev. 1997, 97, 53.

17. The source of oxygen must have been the argon used as "inert" atmospehere. For an account describing unexpected effects due to the presence of oxygen in a reaction carried out under an argon atmosphere, see: Scheiber, S. L.; Ragan, J. A.; Standaert, R. F., in Lindberg, T. (ed.) Strategies and Tactics in Organic Synthesis, vol. 3, chapter 11. Academic Press (San Diego), 1991.

18. Halliwell, B.; Gutteridge, J. M. C. Free Radicals in Biology and Medicine, $3^{\text {rd }}$ Ed. p. 601. Oxford University Press, 1999.

19. (a) Beak, P.; Brubaker, G. R.; Farney, R. F. J. Am. Chem. Soc., 1976, 98, 3621. (b) Wiberg, K. B.; Bailey, W. F. J. Org. Chem. 2002, 67, 5365.

20. (a) Gallina, C.; Liberatori, A. Tetrahedron Lett, 1973, 14, 1135. (b) Gallina, C.; Liberatori, A. Tetrahedron, 1974, 30, 667.

21. However, a detailed study carried out by the Kobayashi group in the context of a total synthesis of neoechinulin A using chiral HPLC as the quantitation method showed a small degree of racemization for this type of reactions. See: Aoki, T. Kamisuki, S.; Kimoto, M.; Ohnishi, K.; Takakusagi, Y.; Kuramochi, K.; Takeda, Y.; Nakazaki, A.; Kuroiwa, K.; Ohuchi, T.; Sugawara, F.; Arai, T.; Kobayashi, S. Synlett 2006, 677.

22. Grahl-Nielsen, O. Tetrahedron Lett. 1969, 10, 2827.

23. There are two previous literature examples of the partial reduction of the benzene ring in pyrazino[2,1-b]quinazoline-3,6-diones, although in an acidic reaction medium. See: (a) Bartolomé, M. T.; Buenadicha, F. L.; Avendaño, C.; Söllhuber, M. Tetrahedron: Asymmetry 1998, 9, 249. (b) Buenadicha, F. L.; Bartolomé, M. T.; Aguirre, M. J.; Avendaño, C.; Söllhuber, M. M. Tetrahedron: Asymmetry 1998, 9, 483.

24. The 2-benzyl and 2-(4-methoxybenzyl) analogues of 4 are known in the literature, and showed similar optical rotation values to our compound (see reference 22b). 